\title{
Development of a Novel Family of Conformationally Restricted Peptides as Potent Nucleators of $\beta$-Turns. Design, Synthesis, Structure and Biological Evaluation of a $\beta$-Lactam Peptide Analogue of Melanostatin.
}

\author{
Claudio Palomo, ${ }^{* \#}$ Jesus M. Aizpurua, ${ }^{* \#}$ Ana Benito, ${ }^{\#}$ José Ignacio Miranda, ${ }^{\#}$ Raluca M. \\ Fratila, ${ }^{\#}$ Carlos Matute, ${ }^{\S}$ Maria Domercq,${ }^{\S}$ Federico Gago,${ }^{\$}$ Sonsoles Martin-Santamaría,${ }^{\$}$ \\ Anthony Linden. ${ }^{\dagger}$
}

Contribution from:

\#Departamento de Química Orgánica I. Facultad de Química. Universidad del País Vasco. Apdo. 1072, 20080 San Sebastian, Spain;

${ }^{\S}$ Departamento de Neurociencias. Facultad de Medicina. Universidad del País Vasco. 48940 Leioa, Spain.

${ }^{\$}$ Departamento de Farmacología. Facultad de Medicina. Universidad de Alcalá de Henares. 28871 Madrid, Spain. (MD calculations)

${ }^{\dagger}$ Organisches-chemisches Institut der Universität Zürich. Winterthurerstrasse 190, CH-8057, Zürich, Switzerland. 
(S) 1-(3-Aza-5-carboxy-4-methyl-2-oxo-pentyl)-3-tertbutoxycarbonylamino-azetidin-2-one<smiles>CC(C)(C)OC(=O)NC1CN(CC(=O)NC(C)(C)C(=O)O)C1=O</smiles>

Reference $\mathrm{N}^{\mathrm{o}}$

8

P. Mol. (g/mol)

329.35

Melt. Point. $\left({ }^{\circ} \mathrm{C}\right)$

128-130 (EtOAc/Hex)

$[\alpha]_{\mathrm{D}}^{25}(\mathrm{c}=0.05, \mathrm{MeOH})$

$-49$

\begin{tabular}{|c|c|c|c|c|c|c|c|c|}
\hline Empirical & \multicolumn{8}{|c|}{ Elemental Analysis } \\
\hline Formula & \multicolumn{3}{|c|}{ Calculated(\%) } & \multicolumn{5}{|c|}{ Found(\%) } \\
\hline $\mathrm{C}_{14} \mathrm{H}_{23} \mathrm{~N}_{3} \mathrm{O}_{6}$ & C 51.05 & H 4.28 & $\mathrm{~N} 12.76$ & C 49.98 & & 4.20 & $\mathrm{~N}$ & 12.78 \\
\hline
\end{tabular}

$\operatorname{IR}\left(\mathrm{cm}^{-1}, \mathrm{KBr}\right)$

$3115,3128,3200(\mathrm{NH}, \mathrm{OH}) ; 1733,1715,1666.7,1691,1666(\mathrm{CO})$

$\mathrm{MS} \mathrm{m} / \mathrm{z}$

58(76); 115(63); 141(100); 169(32); 187(94); 210(64); 228(26); 238(39); 256(24); 284(11); 299(7); $329(\mathrm{M}+)$

\begin{tabular}{|c|c|}
\hline \multicolumn{2}{|l|}{$\left.{ }^{1} \mathrm{H}-\mathrm{RMN}(\delta, \mathrm{ppm})\right) \mathrm{DMSO}_{\mathrm{d} 6}$} \\
\hline $\begin{array}{l}7.72\left(\mathrm{~s}_{\mathrm{s}}, 1 \mathrm{H},(\mathrm{CO}) \mathrm{NHCMe}_{2}\right) \\
5.12\left(\mathrm{~d}_{\mathrm{s}}, 1 \mathrm{H},(\mathrm{NHBoc})\right. \\
4.45\left(\mathrm{~d}, 1 \mathrm{H}, \underline{\mathrm{NCH}}_{2} \mathrm{CONHCMe}\right. \\
\\
4.39\left(\mathrm{~m}, 1 \mathrm{H}, \mathrm{BocNHCHCH}{ }_{2} \mathrm{CON}-\right) \\
3.72\left(\mathrm{~m}, 1 \mathrm{H}, \mathrm{BocNHCHC}{ }_{2} \mathrm{CON}-\right) \\
3.50\left(\mathrm{~m}, 1 \mathrm{H}, \mathrm{BocNHCHC} \underline{\mathrm{H}}_{2} \mathrm{CON}-\right) \\
3.49\left(\mathrm{~d}, 1 \mathrm{H}, \mathrm{NC} \underline{\mathrm{H}}_{2} \mathrm{CONHCMe} \mathrm{CO}_{2} \mathrm{H}\right) \\
\left.1.63(\mathrm{~s}, 3 \mathrm{H}, \mathrm{CO}) \mathrm{NHCMe}_{2} \mathrm{CO}_{2} \mathrm{H}\right) \\
\left.1.58(\mathrm{~s}, 3 \mathrm{H}, \mathrm{CO}) \mathrm{NHCMe}_{2} \mathrm{CO}_{2} \mathrm{H}\right) \\
1.43(\mathrm{~s}, 9 \mathrm{H}, \mathrm{CMe} \\
)\end{array}$ & $\begin{array}{l}\mathrm{J}=4.0 \mathrm{~Hz} \\
\mathrm{~J}=16.0 \mathrm{~Hz}\end{array}$ \\
\hline
\end{tabular}

\begin{tabular}{|c|}
\hline${ }^{13} \mathrm{C}-\mathrm{RMN}(\delta, \mathrm{ppm}) \mathrm{DMSO}_{\mathrm{d} 6}$ \\
\hline 175.1 \\
167.3 \\
166.2 \\
154.8 \\
78.8 \\
57.5 \\
55.1 \\
47.9 \\
44.1 \\
28.1 \\
25.0 \\
24.9
\end{tabular}

Comments: 
(R)-1-(3-Aza-4-benzyloxycarbonyl-2-oxo-butyl)-3-benzyl-3-[1-aza-

(3-tert-butoxycarbonylamino-2-oxo-propyl]-azetidin-2-one.

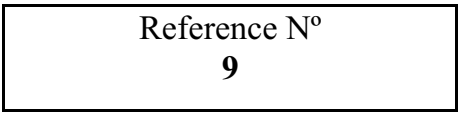<smiles>CC(C)(C)OC(=O)NCC(=O)N[C@]1(Cc2ccccc2)CN(CC(=O)NCC(=O)OCc2ccccc2)C1=O</smiles>

\begin{tabular}{|c|}
\hline M.W $(\mathrm{g} / \mathrm{mol})$ \\
538.59 \\
\hline Melting Point $\left({ }^{\circ} \mathrm{C}\right)$ \\
$50-60$ \\
\hline$[\alpha]_{\mathrm{D}}^{25}\left(\mathrm{c}=1.0, \mathrm{Cl}_{2} \mathrm{CH}_{2}\right)$ \\
+63.9 \\
\hline
\end{tabular}

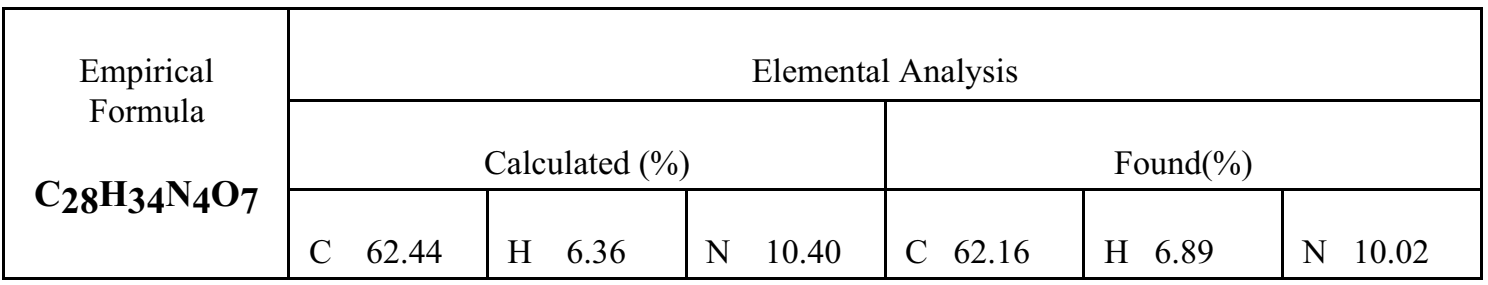

$\operatorname{IR}\left(\mathrm{cm}^{-1}, \mathrm{KBr}\right)$

$\mathrm{MS} \mathrm{m} / \mathrm{z}$

${ }^{1} \mathrm{H}-\mathrm{NMR}(\delta, \mathrm{ppm}) \mathrm{CDCl}_{3}$

$8.16\left(\mathrm{t}_{\mathrm{s}}, 1 \mathrm{H},(\mathrm{CO}) \mathrm{NHCH}_{2} \mathrm{CO}_{2} \mathrm{Bn}\right)$

7.39-7.31 (m, 10H, Ar)

$6.65(\mathrm{~s}, 1 \mathrm{H},(\mathrm{CO}) \mathrm{N} \underline{\mathrm{HC}})$

$5.14\left(2 \mathrm{~d}, 2 \mathrm{H},-\mathrm{OC} \underline{\mathrm{H}_{2}} \mathrm{Ph}\right)$

4.97 (s, 1H, BocN $\underline{\mathrm{H}})$

$4.38\left(\mathrm{~d}, 1 \mathrm{H},=\mathrm{NCH}_{2} \mathrm{CONHCH}_{2}-\right)$

$\mathrm{J}=17.6 \mathrm{~Hz}$

$4.30\left(\mathrm{dd}, 1 \mathrm{H},-\mathrm{NHCH}_{2} \mathrm{CO}_{2} \mathrm{Bn}\right)$

$\mathrm{J}=17.6,7.0 \mathrm{~Hz}$

3.89-3.78 (m, 3H,- $\left.\mathrm{CCH}_{2} \mathrm{~N}, \mathrm{NHCH}_{2} \mathrm{CO}_{2} \mathrm{Bn}, \mathrm{BocNHCH}_{2}\right)$

$3.65\left(\mathrm{dd}, 1 \mathrm{H}, \mathrm{BocNHCH} \underline{\mathrm{H}}_{2}-\right)$

$3.47\left(\mathrm{~d}, 1 \mathrm{H},-\mathrm{NCH}_{2} \mathrm{CONHCH}_{2}-\right)$

$3.42\left(\mathrm{~d}, 1 \mathrm{H},-\mathrm{CC} \underline{\mathrm{H}}_{2} \mathrm{~N}-\right)$

$3.20\left(\mathrm{~d}, 1 \mathrm{H},-\mathrm{OC} \underline{\mathrm{H}}_{2} \mathrm{Ph}\right)$

$3.12\left(\mathrm{~d}, 1 \mathrm{H},-\mathrm{OC} \underline{\mathrm{H}}_{2} \mathrm{Ph}\right)$

$\mathrm{J}=17.0,6.0 \mathrm{~Hz}$

$\mathrm{J}=17.6 \mathrm{~Hz}$

$\mathrm{J}=5.6 \mathrm{~Hz}$

$\mathrm{J}=14.1 \mathrm{~Hz}$

$\mathrm{J}=14.3 \mathrm{~Hz}$

\begin{tabular}{|cc|}
\hline${ }^{13} \mathrm{C}-\mathrm{NMR}(\delta, \mathrm{ppm}) \mathrm{CDCl}_{3}$ \\
\hline 170.2 & 45.0 \\
169.4 & 44.5 \\
167.9 & 41.1 \\
167.8 & 39.3 \\
135.4 & 28.3 \\
133.5 & \\
129.7 & \\
129.1 & \\
128.6 & \\
128.4 & \\
127.9 & \\
80.8 & \\
68.2 & \\
66.9 & \\
51.4 & \\
& \\
\end{tabular}

Comments: Analytical sample obtained by preparative HPLC 
(3R,4R)-1-[3-Aza-4-carboxy-4-methyl-2-oxo-pentyl]-3-benzyl-3tert-butoxicarbonylamino-4-isobutylazetidin-2-one.

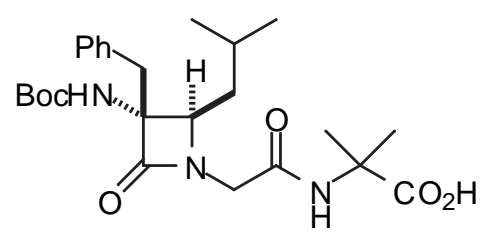

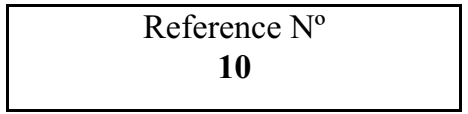

\begin{tabular}{|c|}
\hline M.W $(\mathrm{g} / \mathrm{mol})$ \\
475.59 \\
\hline Melting Point $\left({ }^{\circ} \mathrm{C}\right)$ \\
$129-130$ \\
\hline$[\alpha]_{\mathrm{D}}^{25}\left(\mathrm{c}=1.0, \mathrm{Cl}_{2} \mathrm{CH}_{2}\right)$ \\
+76.7 \\
\hline
\end{tabular}

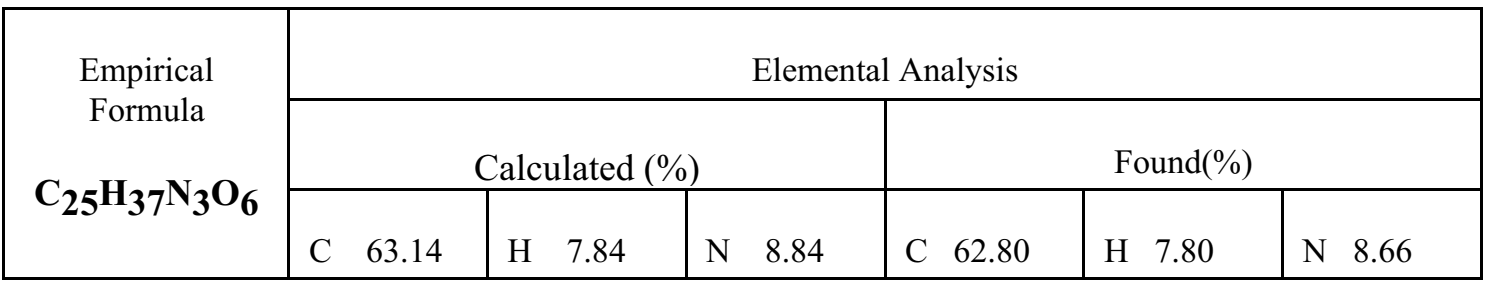

$\operatorname{IR}\left(\mathrm{cm}^{-1}\right.$, film $) \quad 3315.0 ; 3120.0 ; 2946.0 ; 1741.1 ; 1717.7 ; 1699.8 ; 1690.7$

\begin{tabular}{|c|l|}
\hline HPLC-MS & $-\mathrm{MS}: 474.3$ \\
Ion Source Type: & $-\mathrm{MS} 2(474.3): 400.2$ \\
ESI* & $-\mathrm{MS} 3(400.2): 357.2,128.3$ \\
& $-\mathrm{MS} 4(357.2): 339.0,313.1,256.1,228.0,185.0,141.0,128.0$ \\
\hline
\end{tabular}

${ }^{1} \mathrm{H}-\mathrm{NMR}(\delta, \mathrm{ppm}) \mathrm{CDCl}_{3}$

$7.79(\mathrm{~s}, 1 \mathrm{H}, \mathrm{NH})$

7.31-7.19 (m, 5H, Ar)

4.63 (s, 1H, NHBoc)

$4.42\left(\mathrm{~d}, 1 \mathrm{H}, \mathrm{NC}_{2} \mathrm{C}=\mathrm{O}\right)$

4.23- $4.20\left(\mathrm{~m}, 1 \mathrm{H}, \underline{\mathrm{HC}}-{ }^{\mathrm{i}} \mathrm{Bu}\right)$

$3.50\left(\mathrm{~d}, 1 \mathrm{H}, \mathrm{NCH}_{2} \mathrm{C}=\mathrm{O}\right)$

$3.15\left(\mathrm{~d}, 1 \mathrm{H}, \mathrm{CH}_{2} \mathrm{Ph}\right)$

$2.87\left(\mathrm{~d}, 1 \mathrm{H}, \mathrm{CH}_{2} \mathrm{Ph}\right)$

1.67-1.63 (m, 1H, $\left.\underline{\mathrm{H}}\left(\mathrm{CH}_{3}\right)_{2}\right)$

$1.54\left(\mathrm{~s}, 3 \mathrm{H}, \mathrm{HNC}\left(\mathrm{CH}_{3}\right)_{2} \mathrm{C}=\mathrm{O}\right)$

$1.52\left(\mathrm{~s}, 3 \mathrm{H}, \mathrm{HNC}\left(\mathrm{CH}_{3}\right)_{2} \mathrm{C}=\mathrm{O}\right)$

1.53-1.51 (m, 2H, $\left.\underline{\mathrm{C}}_{2} \mathrm{CH}\left(\mathrm{CH}_{3}\right)_{2}\right)$

1.31 (s, 9H, Boc)

0.93 (s, 3H, $\left.\mathrm{CH}_{2} \mathrm{CH}\left(\mathrm{CH}_{3}\right)_{2}\right)$

0.92 (s, 3H, $\left.\mathrm{CH}_{2} \mathrm{CH}\left(\mathrm{C}_{3}\right)_{2}\right)$

\begin{tabular}{|rr|}
\hline${ }^{13} \mathrm{C}-\mathrm{NMR}(\delta, \mathrm{ppm}) \mathrm{CDCl}_{3}$ \\
\hline & \\
177.6 & 28.0 \\
168.9 & 26.1 \\
167.5 & 25.4 \\
154.5 & 24.4 \\
133.7 & 23.1 \\
130.0 & 22.3 \\
128.7 & \\
127.4 & \\
80.8 & \\
68.6 & \\
61.0 & \\
56.5 & \\
43.5 & \\
36.5 & \\
36.4 & \\
& \\
\hline
\end{tabular}

Comments: Analytical sample by preparative HPLC. Column: Lichrosorb Si60 (7 $\mu \mathrm{m})$ 250x25mm; Eluant: EtOAc/AcOH(0.5\%); r.t.: 15 min

$* \mathrm{MeCN} / \mathrm{NH}_{3}$, negative polarity 
(3S,4R)-1-[3-Aza-4-carboxy-4-methyl-2-oxo-pentyl]-3-benzyl-3-

tert-butoxicarbonylamino-4-isobutylazetidin-2-one.
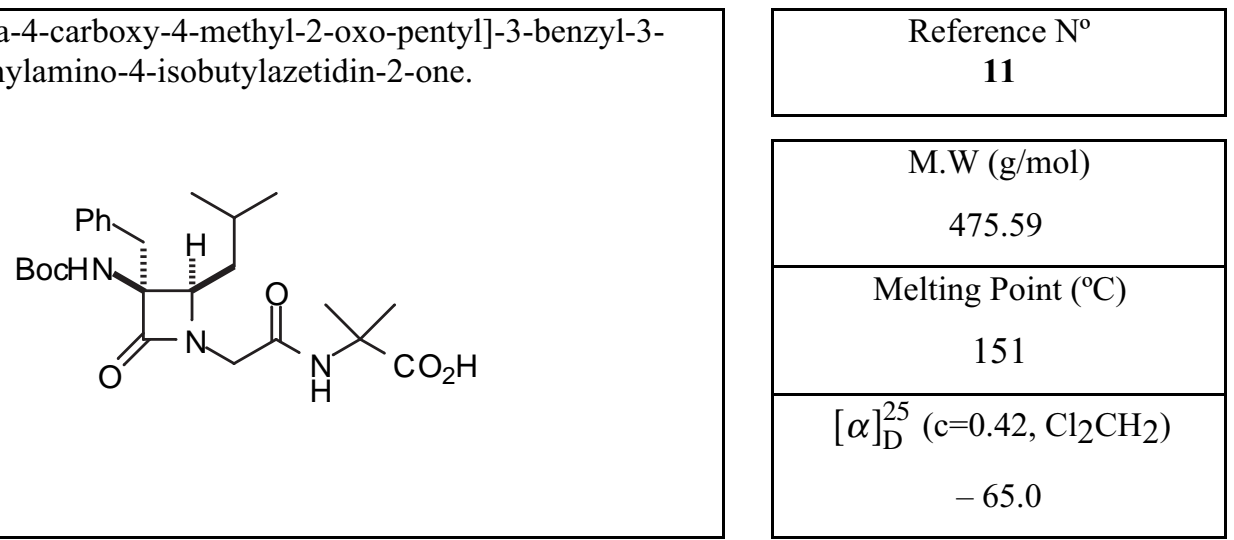

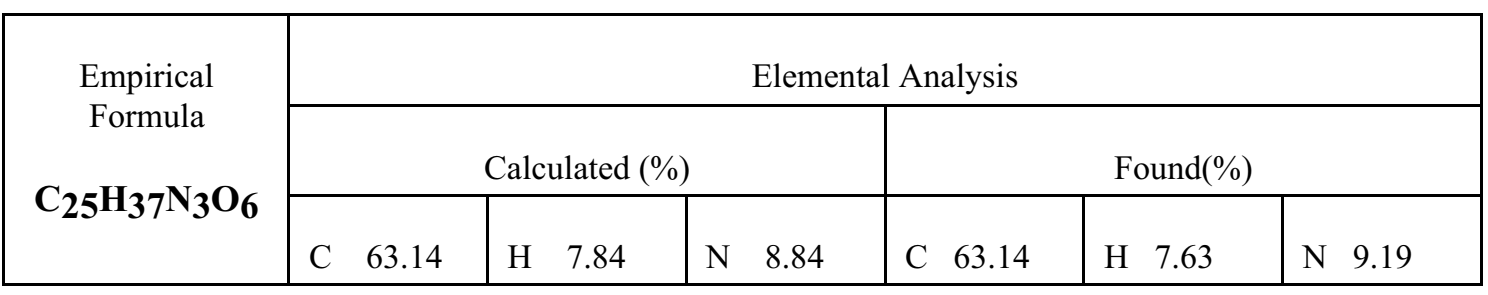

$\operatorname{IR}\left(\mathrm{cm}^{-1}\right.$, film $)$

$3322.8 ; 2959.8 ; 2932.7 ; 1760.8 ; 1716.4 ; 1698.7 ; 1661.0 ; 1654.0$

\begin{tabular}{c|l}
\hline HPLC-MS & -MS: 474.3 \\
Ion Source Type: & $-\mathrm{MS} 2(474.3): 400.2$ \\
ESI* $^{*}$ & $-\mathrm{MS} 3(400.2): 357.1,297.2,253.2,227.2,199.1,128.1$ \\
& $-\mathrm{MS} 4(357.2): 339.1,313.2,228.0,185.1,141.0,128.0$
\end{tabular}

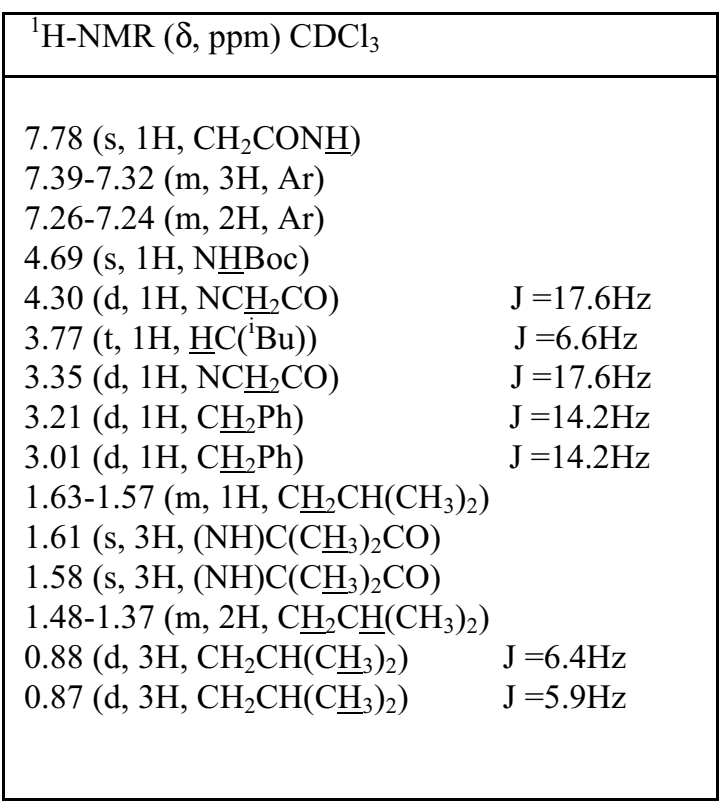

\begin{tabular}{|cc|}
\hline${ }^{13} \mathrm{C}-\mathrm{NMR}(\delta, \mathrm{ppm}) \mathrm{CDCl}_{3}$ \\
\hline & \\
176.9 & 28.1 \\
168.7 & 25.5 \\
168.2 & 24.4 \\
154.8 & 22.9 \\
134.0 & 22.6 \\
129.8 & \\
129.0 & \\
127.8 & \\
80.8 & \\
70.2 & \\
64.3 & \\
56.7 & \\
44.9 & \\
40.4 & \\
36.6 & \\
& \\
\end{tabular}

Comments: $* \mathrm{MeCN} / \mathrm{NH}_{3}$, negative polarity 
(3R) 1-Amidomethyl-3-isobutyl-3-[(2S)-pyrrolidin-2-ylcarbonylamino]azetidin-2-one.
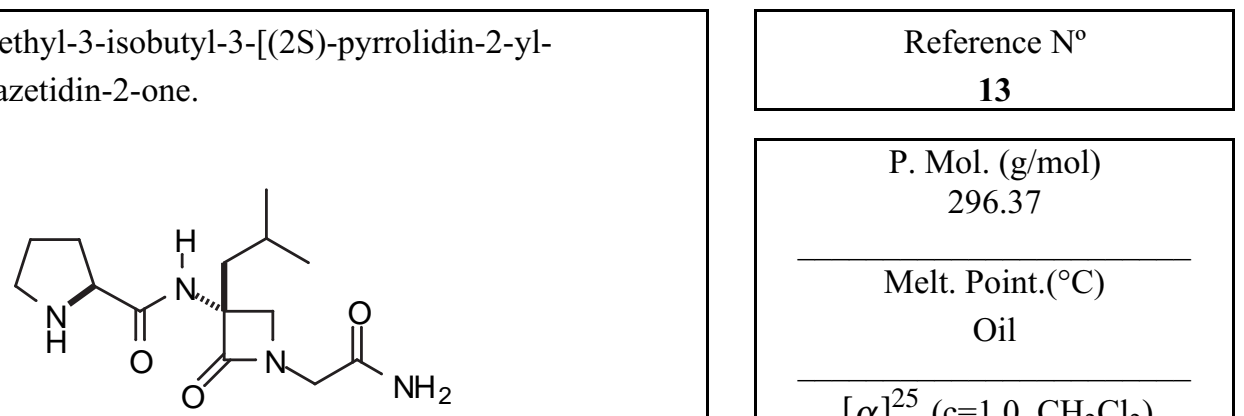

Pol. $(\mathrm{g} / \mathrm{mol})$

296.37

Melt. Point. $\left({ }^{\circ} \mathrm{C}\right)$

Oil

$[\alpha]_{\mathrm{D}}^{25}\left(\mathrm{c}=1.0, \mathrm{CH}_{2} \mathrm{Cl}_{2}\right)$
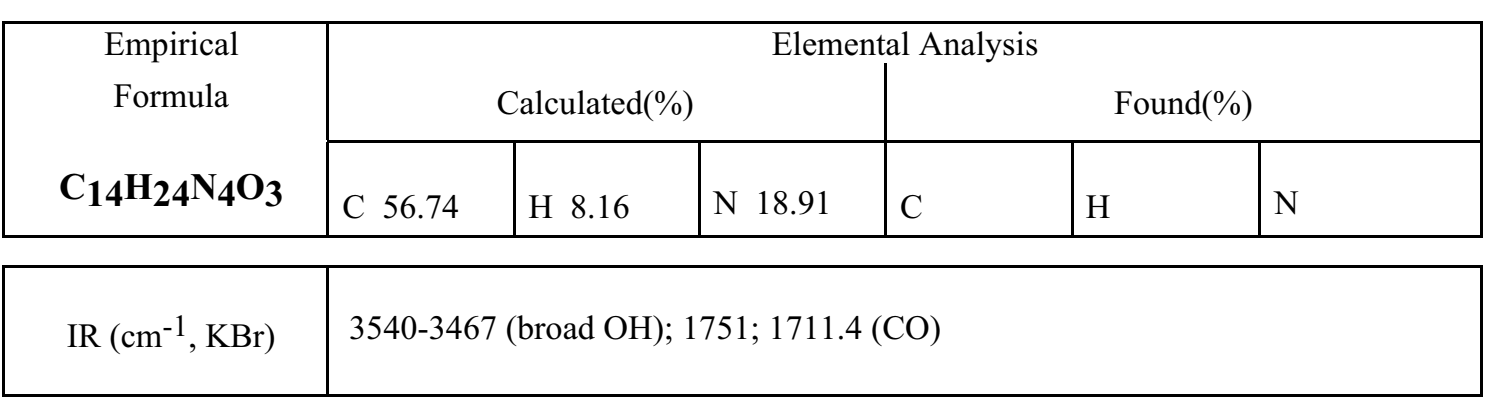

\begin{tabular}{|c|l|}
\hline HPLC- MS & + MS: 297.3 \\
Ion Source Type: & + MS2(297.3): 228.1, 182.1 \\
ESI & + MS3(228.1): 182.1 \\
\hline
\end{tabular}

\begin{tabular}{|c|c|c|}
\hline${ }^{1} \mathrm{H}-\mathrm{RMN}(\delta, \mathrm{ppm}) \mathrm{CD}_{3} \mathrm{OD}$ & ${ }^{1} \mathrm{H}-\mathrm{NMR}(\delta, \mathrm{ppm})$ DMSOd6 & ${ }^{13} \mathrm{C}-\mathrm{RMN} \mathrm{CD}{ }_{3} \mathrm{OD}$ \\
\hline $\begin{array}{ll}4.19\left(\mathrm{~d}, 1 \mathrm{H}, \mathrm{CH}_{2} \mathrm{CONH}_{2}\right) & \mathrm{J}=17.6 \mathrm{~Hz} \\
3.81\left(\mathrm{~d}, 1 \mathrm{H},(\mathrm{CO}) \mathrm{NCH}_{2}\right) & \mathrm{J}=4.4 \mathrm{~Hz} \\
3.69(\mathrm{~m}, 1 \mathrm{H}, \mathrm{CH}(\mathrm{Pro})) & \\
3.69\left(\mathrm{~d}, 1 \mathrm{H}, \mathrm{CH}_{2} \mathrm{CONH}\right. & \\
3.50\left(\mathrm{~d}, 1 \mathrm{H},(\mathrm{CO}) \mathrm{NCH}_{2}\right) & \mathrm{J}=4.4 \mathrm{~Hz} \\
2.96-2.93\left(\mathrm{~m}, 2 \mathrm{H}, \mathrm{HNC} \underline{H}_{2}\right) & \\
2.15-2.11(\mathrm{~m}, 1 \mathrm{H}, \mathrm{Pro}) & \\
1.95-1.73\left(\mathrm{~m}, 6 \mathrm{H},{ }^{\mathrm{i}} \mathrm{Bu} ; \mathrm{Pro}\right) & \\
1.03\left(\mathrm{~d}, 3 \mathrm{H}, \mathrm{CH}_{2} \mathrm{CH}\left(\mathrm{CH}_{3}\right)_{2}\right) \mathrm{J}=6.4 \mathrm{~Hz} \\
0.99 \mathrm{~J}=6.4 \mathrm{~Hz}\left(\mathrm{~d}, 3 \mathrm{H}, \underline{\mathrm{CH}}_{2} \mathrm{CH}\left(\underline{\mathrm{CH}}_{3}\right)_{2}\right)\end{array}$ & $\begin{array}{l}8.23(\mathrm{~s}, 1 \mathrm{H}, \mathrm{NH}) \\
7.75(\mathrm{~s}, 1 \mathrm{H}, \mathrm{NH}) \\
7.31(\mathrm{~s}, 1 \mathrm{H}, \mathrm{NH}) \\
3.90\left(\mathrm{~d}, 1 \mathrm{H}, \mathrm{CH}_{2} \mathrm{CONH}_{2}\right) \mathrm{J}=17.6 \mathrm{~Hz} \\
3.62-3.53(\mathrm{~m}, 3 \mathrm{H}) \\
3.36\left(\mathrm{~d}, 1 \mathrm{H},(\mathrm{CO}) \mathrm{NCH}_{2}\right) \quad \mathrm{J}=4.89 \mathrm{~Hz} \\
2.93-2.85(\mathrm{~m}, 1 \mathrm{H}, \mathrm{Pro}) \\
2.77-2.71(\mathrm{~m}, 1 \mathrm{H}, \mathrm{Pro}) \\
1.99-1.57\left(\mathrm{~m}, 6 \mathrm{H},{ }_{\mathrm{i}} \mathrm{Bu} ; \mathrm{Pro}\right) \\
0.96\left(\mathrm{~d}, 3 \mathrm{H}, \mathrm{CH}_{2} \mathrm{CH}\left(\mathrm{CH}_{3}\right)_{2}\right) \mathrm{J}=6.4 \mathrm{~Hz} \\
0.91\left(\mathrm{~d}, 3 \mathrm{H}, \mathrm{CH}_{2} \mathrm{CH}\left(\mathrm{CH}_{3}\right)_{2}\right) \mathrm{J}=6.4 \mathrm{~Hz}\end{array}$ & $\begin{array}{r}177.5 \\
172.9 \\
171.3 \\
68.8 \\
61.4 \\
54.6 \\
45.4 \\
43.0 \\
31.9 \\
27.1 \\
25.8 \\
24.3 \\
23.2\end{array}$ \\
\hline
\end{tabular}

Comments: : * $\mathrm{MeCN} / \mathrm{NH}_{3}$, positive polarity 
(S) 3-tert-Butoxycarbonylamino-1-carboxymethylazetidin-2-one
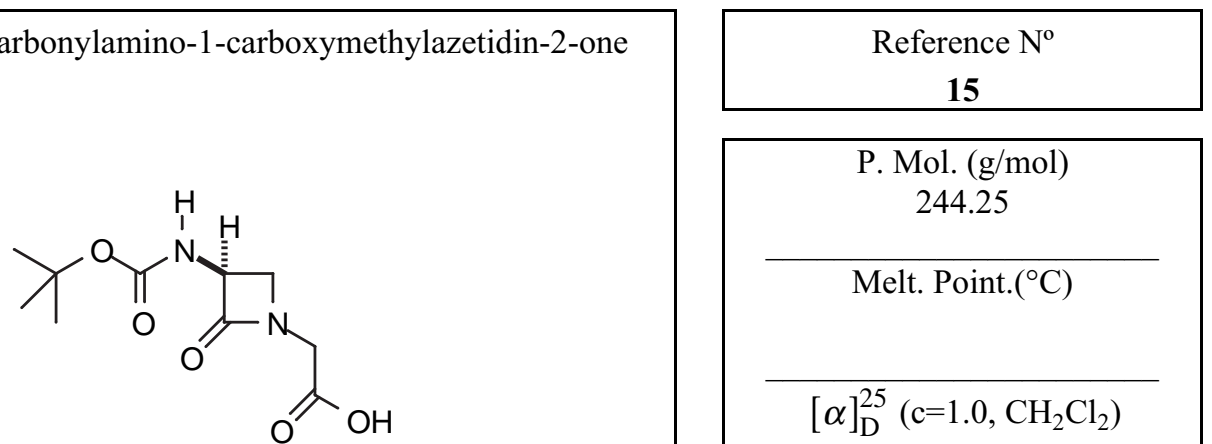

P. Mol. $(\mathrm{g} / \mathrm{mol})$

244.25

Melt. Point. $\left({ }^{\circ} \mathrm{C}\right)$

$[\alpha]_{\mathrm{D}}^{25}\left(\mathrm{c}=1.0, \mathrm{CH}_{2} \mathrm{Cl}_{2}\right)$

\begin{tabular}{|c|c|c|c|c|c|c|c|c|c|}
\hline Empirical & \multicolumn{9}{|c|}{ Elemental Analysis } \\
\hline Formula & \multicolumn{4}{|c|}{ Calculated(\%) } & \multicolumn{5}{|c|}{ Found(\%) } \\
\hline $\mathrm{C}_{10} \mathrm{H}_{16} \mathrm{~N}_{2} \mathrm{O}_{5}$ & C 36.47 & H 4.90 & & 8.50 & C 36.81 & & 4.9 & $\mathrm{~N}$ & 8.59 \\
\hline
\end{tabular}

$\operatorname{IR}\left(\mathrm{cm}^{-1}, \mathrm{KBr}\right)$ 3540-3467 (broad OH); 1751; 1711.4 (CO)

MS m/z

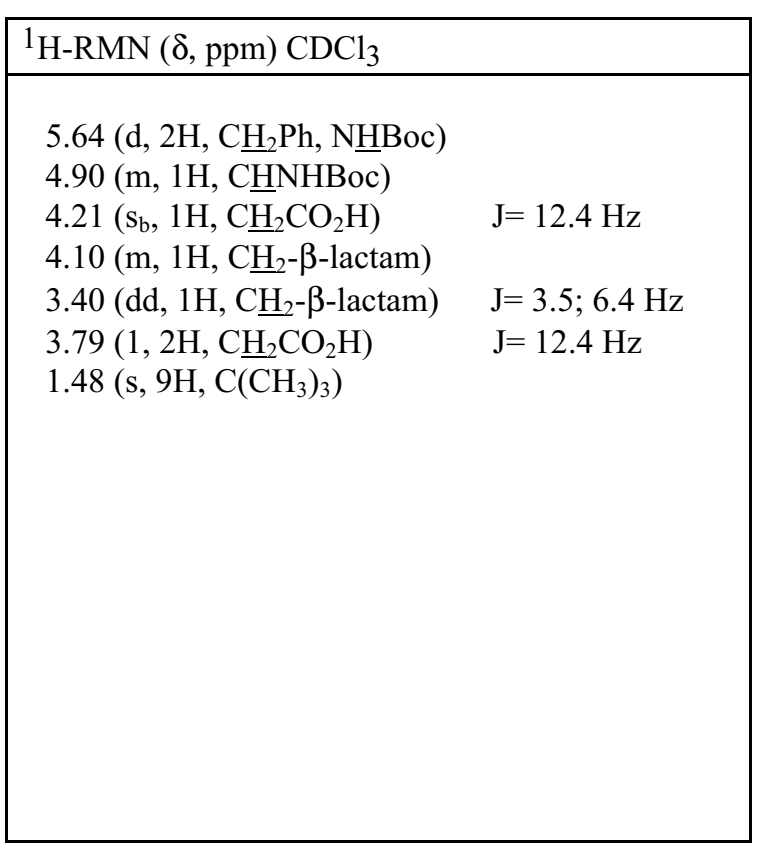

\begin{tabular}{|l|}
\hline${ }^{13} \mathrm{C}-\mathrm{RMN}(\delta, \mathrm{ppm}) \mathrm{CDCl}_{3}$ \\
\hline \\
171.2 \\
168.1 \\
155.5 \\
67.5 \\
58.2 \\
50.6 \\
42.1 \\
28.9 \\
\\
\end{tabular}

Comments: 
(R)-1-(3-Aza-4-benzyloxycarbonyl-2-oxo-butyl)-3-benzyl-3-(2-

tert-butoxycarbonylamino-azetidin-2-one.
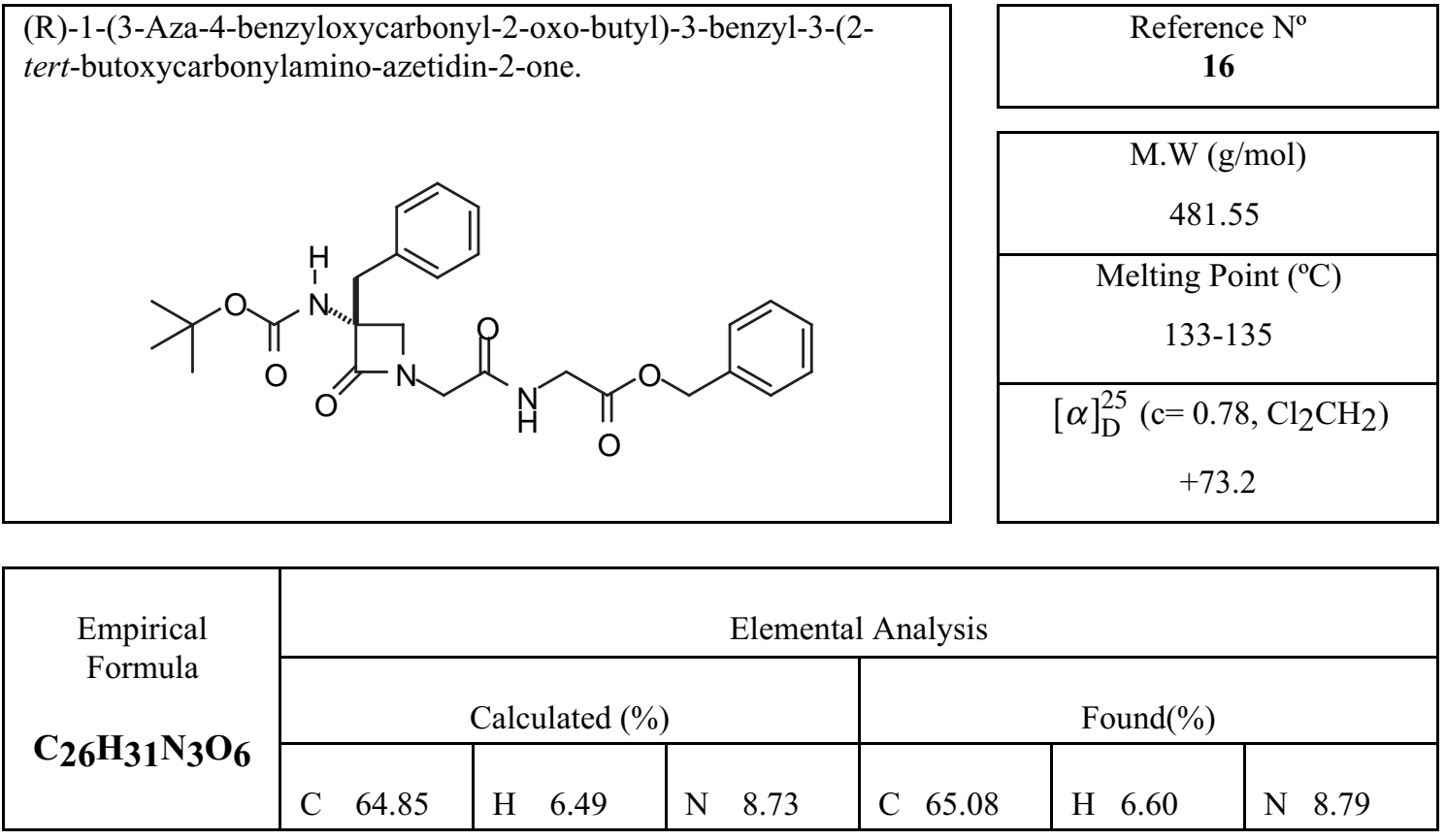

$\operatorname{IR}\left(\mathrm{cm}^{-1}, \mathrm{KBr}\right) \quad 3310.8 ; 3265.9(\mathrm{NH}) .1755 .2 ; 1766.9 ; 1694.2 ; 1684.5(\mathrm{C}=\mathrm{O})$

$\mathrm{MS} \mathrm{m} / \mathrm{z}$

${ }^{1} \mathrm{H}-\mathrm{NMR}(\delta, \mathrm{ppm}) \mathrm{CDCl}_{3}$

$8.30(\mathrm{~s}, 1 \mathrm{H}, \mathrm{NH})$

7.39-7.25 (m, 10H, Ar)

$5.13\left(2 \mathrm{~d}, 2 \mathrm{H},-\mathrm{OCH}_{2} \mathrm{Ph}\right)$

$4.80(\mathrm{~s}, 1 \mathrm{H}, \mathrm{BocN} \underline{\mathrm{H}})$

$4.40\left(\mathrm{~d}, 1 \mathrm{H}, \mathrm{NCH}_{2} \mathrm{CONH}\right)$

$\mathrm{J}=17.6 \mathrm{~Hz}$

$4.40\left(\mathrm{dd}, 1 \mathrm{H}, \mathrm{NHCH}_{2} \mathrm{COOBn}\right)$

$\mathrm{J}=17.7 \mathrm{~Hz}$

$3.85\left(\mathrm{~d}, 1 \mathrm{H}, \mathrm{CC}_{2} \mathrm{~N}\right)$

$\mathrm{J}=5.5 \mathrm{~Hz}$

$3.75\left(\mathrm{dd}, 1 \mathrm{H},-\mathrm{NHCH}_{2} \mathrm{COOBn}\right) \quad \mathrm{J}=17.5 ; 4.5 \mathrm{~Hz}$

3.45-3.40 (m, $\left.2 \mathrm{H}, \mathrm{CC}_{2} \mathrm{~N} ; \mathrm{NC}_{2} \mathrm{CONH}\right)$

$3.19\left(\mathrm{~d}, 1 \mathrm{H},-\mathrm{CH}_{2} \mathrm{Ph}\right)$

$\mathrm{J}=14.3 \mathrm{~Hz}$

$3.06\left(\mathrm{~d}, 1 \mathrm{H},-\underline{\mathrm{CH}}_{2} \mathrm{Ph}\right)$

$\mathrm{J}=14.3 \mathrm{~Hz}$

$1.39\left(\mathrm{~s}, 9 \mathrm{H},-\mathrm{OCOC}\left(\mathrm{CH}_{3}\right)_{3}\right)$

\begin{tabular}{|cc|}
\hline${ }^{13} \mathrm{C}-\mathrm{NMR}(\delta, \mathrm{ppm}) \mathrm{CDCl}_{3}$ \\
\hline & \\
169.4 & 40.8 \\
168.5 & 39.5 \\
167.9 & 28.2 \\
154.6 & \\
135.4 & \\
133.7 & \\
129.7 & \\
129.0 & \\
128.6 & \\
128.4 & \\
128.3 & \\
127.8 & \\
81.5 & \\
68.6 & \\
66.9 & \\
52.1 & \\
45.0 & \\
\hline
\end{tabular}

Comments: 
(3R,4R)-3-Benzyl-1-[bis(trimethylsilyl)methyl]-4-isobutyl-3-

[(4S)-4-phenyl-2-oxo-oxazolidin-3-yl]-azetidin-2-one
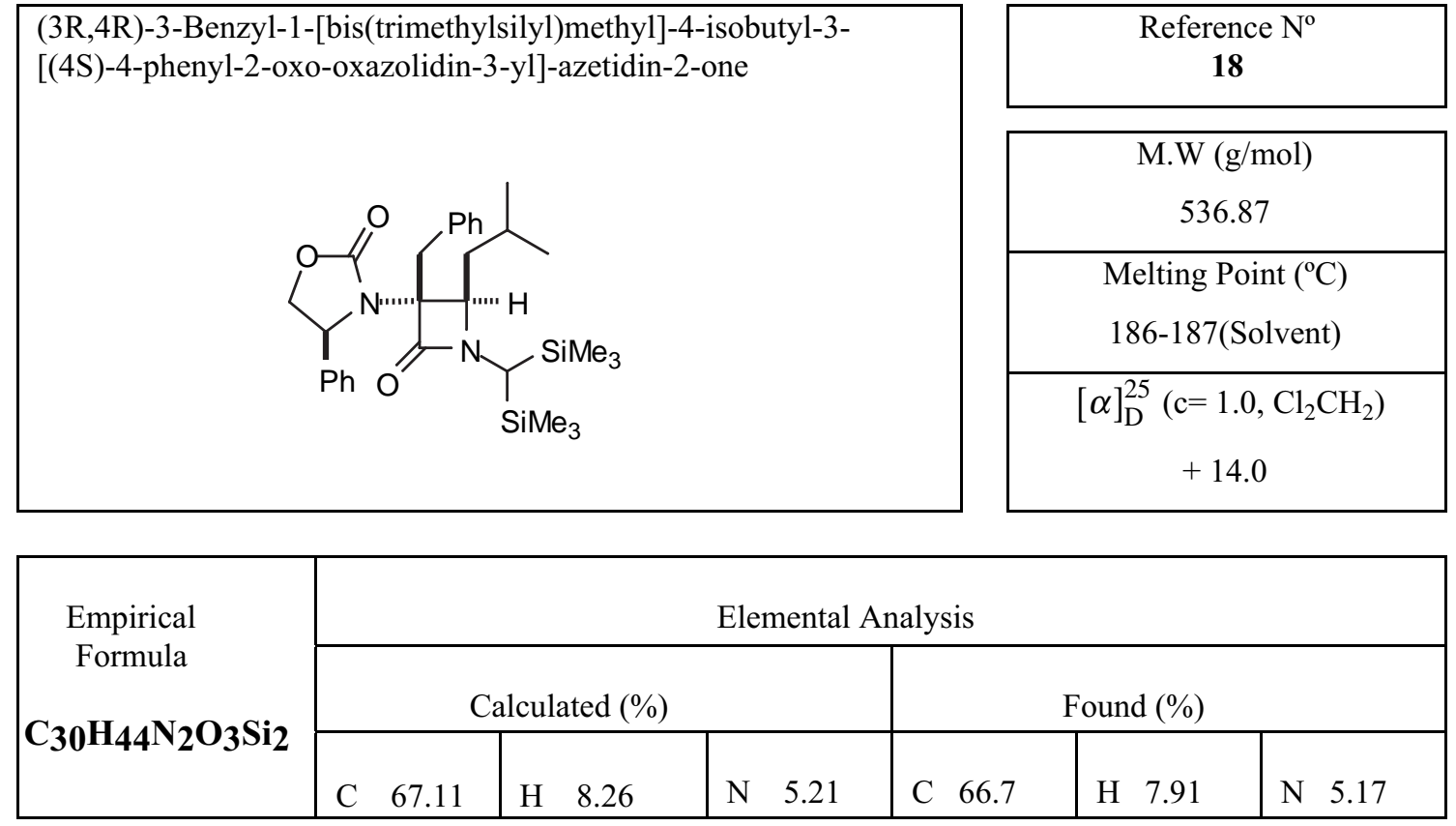

\begin{tabular}{|l|l}
\hline $\mathrm{IR}\left(\mathrm{cm}^{-1}, \mathrm{KBr}\right)$ & $1752 ; 1741(\mathrm{C}=\mathrm{O})$
\end{tabular}

MS m/z

73(36); 91(29); 104(63); 278(30); 279(86); 292(82); 293(35); 335(100); $521(10) ; 536(6)$

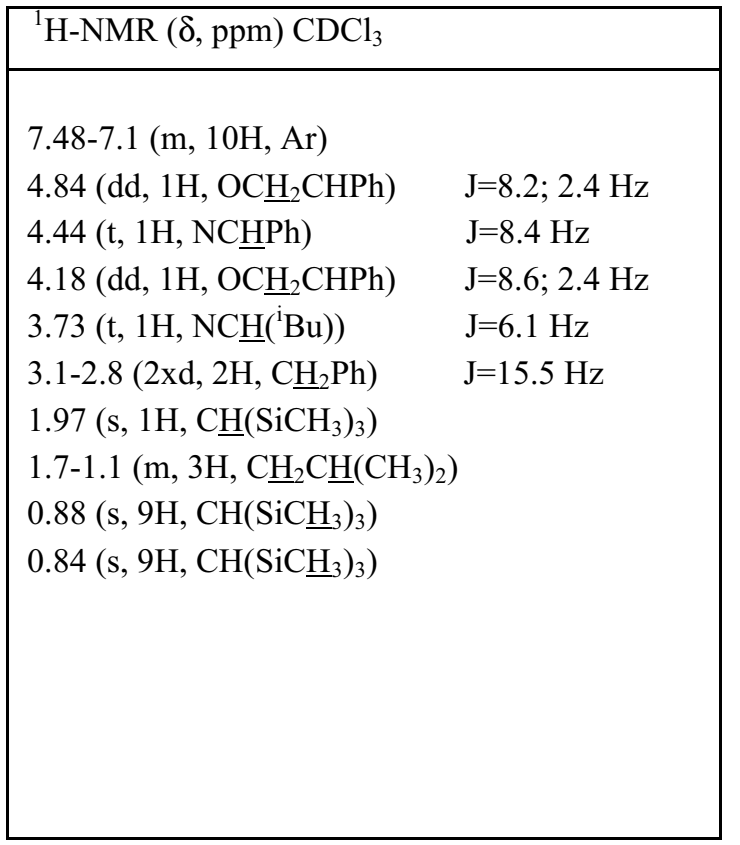

\begin{tabular}{|cc|}
\hline${ }^{13} \mathrm{C}-\mathrm{NMR}\left(\delta,{\mathrm{ppm}) \mathrm{CDCl}_{3}}\right.$ \\
\hline 165.5 & 22.9 \\
157.6 & 0.85 \\
140.9 & 0.65 \\
136.5 & \\
130.9 & \\
129.8 & \\
129.4 & \\
128.9 & \\
127.7 & \\
127.2 & \\
71.7 & \\
65.9 & \\
59.9 & \\
38.3 & \\
36.6 & \\
26.2 & \\
24.0 & \\
\end{tabular}

Comments: 
(3R,4R)-3-Benzyl-3-tert-butoxycarbonylamino-4-isobutylazetidin-2-one

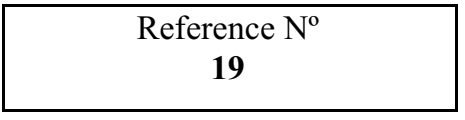<smiles>CC(C)CC1NC(=O)[C@]1(Cc1ccccc1)NC(=O)OC(C)(C)C</smiles>

\begin{tabular}{|c|}
\hline M.W $(\mathrm{g} / \mathrm{mol})$ \\
332.44 \\
\hline Melting Point $\left({ }^{\circ} \mathrm{C}\right)$ \\
$53-55$ \\
\hline$[\alpha]_{\mathrm{D}}^{25}\left(\mathrm{c}=1.0, \mathrm{Cl}_{2} \mathrm{CH}_{2}\right)$ \\
+78.3 \\
\hline
\end{tabular}

\begin{tabular}{|c|c|c|c|c|c|c|c|c|c|}
\hline \multirow{3}{*}{$\begin{array}{c}\text { Empirical } \\
\text { Formula } \\
\mathrm{C}_{\mathbf{1 9}} \mathrm{H}_{\mathbf{2 8}} \mathbf{N}_{2} \mathrm{O}_{3}\end{array}$} & \multicolumn{9}{|c|}{ Elemental Analysis } \\
\hline & \multicolumn{6}{|c|}{ Calculated (\%) } & \multicolumn{3}{|c|}{ Found $(\%)$} \\
\hline & & 68.65 & & 8.49 & & 8.43 & C 68.73 & H 8.56 & $\mathrm{~N} \quad 8.40$ \\
\hline
\end{tabular}

\section{$\operatorname{IR}\left(\mathrm{cm}^{-1}, \mathrm{KBr}\right) \quad 3270.0(\mathrm{~N}-\mathrm{H}) ; 2950.5 ; 1760.9 ; 1708.9(\mathrm{C}=\mathrm{O})$}

MS m/z

57(18), 91(41), 129(61), 146(56), 177(95), 233(100), 259(9), 289(16)

\begin{tabular}{|ll|}
\hline${ }^{1} \mathrm{H}-\mathrm{NMR}(\delta, \mathrm{ppm}) \mathrm{CDCl}_{3}$ & \\
$7.31-7.21(\mathrm{~m}, 5 \mathrm{H}, \mathrm{Ar})$ & \\
$6.55\left(\mathrm{~s}_{\mathrm{b}}, 1 \mathrm{H}, \mathrm{NH}\right)$ & \\
$4.97\left(\mathrm{~s}_{\mathrm{b}}, 1 \mathrm{H}, \mathrm{N} \underline{\mathrm{HBoc}}\right)$ & $\mathrm{J}=9.2 \mathrm{~Hz}$ \\
$3.93(\mathrm{~d}, 1 \mathrm{H}, \mathrm{CC} \underline{\mathrm{H}})$ & $\mathrm{J}=13.7 \mathrm{~Hz}$ \\
$3.26\left(\mathrm{~d}, 1 \mathrm{H}, \mathrm{CH}_{2} \mathrm{Ph}\right)$ & $\mathrm{J}=14.0 \mathrm{~Hz}$ \\
$3.04\left(\mathrm{~d}, 1 \mathrm{H}, \mathrm{C}_{2} \mathrm{Ph}\right)$ & \\
$1.90-1.68\left(\mathrm{~m}, \underline{\mathrm{H}}, \underline{\mathrm{H}}_{2} \mathrm{C} \underline{\mathrm{H}}\left(\mathrm{CH}_{3}\right)_{2}\right)$ & \\
$1.50-1.45\left(\mathrm{~m}, 1 \mathrm{H}, \underline{\mathrm{H}}_{2} \mathrm{C} \underline{\mathrm{H}}\left(\mathrm{CH}_{3}\right)_{2}\right)$ & \\
$1.41\left(\mathrm{~s}, 9 \mathrm{H}, \mathrm{Boc}^{2}\right)$ & \\
$0.98\left(\mathrm{~s}, 3 \mathrm{H}, \mathrm{CH}_{3}\right)$ & \\
$0.96\left(\mathrm{~s}, 3 \mathrm{H}, \mathrm{CH}_{3}\right)$ & \\
& \\
& \\
& \\
\end{tabular}

\begin{tabular}{|c|}
\hline${ }^{13} \mathrm{C}-\mathrm{NMR}(\delta, \mathrm{ppm}) \mathrm{CDCl}_{3}$ \\
\hline 169.0 \\
154.5 \\
135.3 \\
130.4 \\
128.3 \\
126.9 \\
80.0 \\
69.4 \\
59.7 \\
39.5 \\
35.3 \\
28.3 \\
26.3 \\
23.2 \\
22.1 \\
\end{tabular}

Comments: 
(3R, 4R)-3-Benzyl-3-tert-butoxycarbonylamino-1-carboxymethyl-

4-isobutylazetidin-2-one
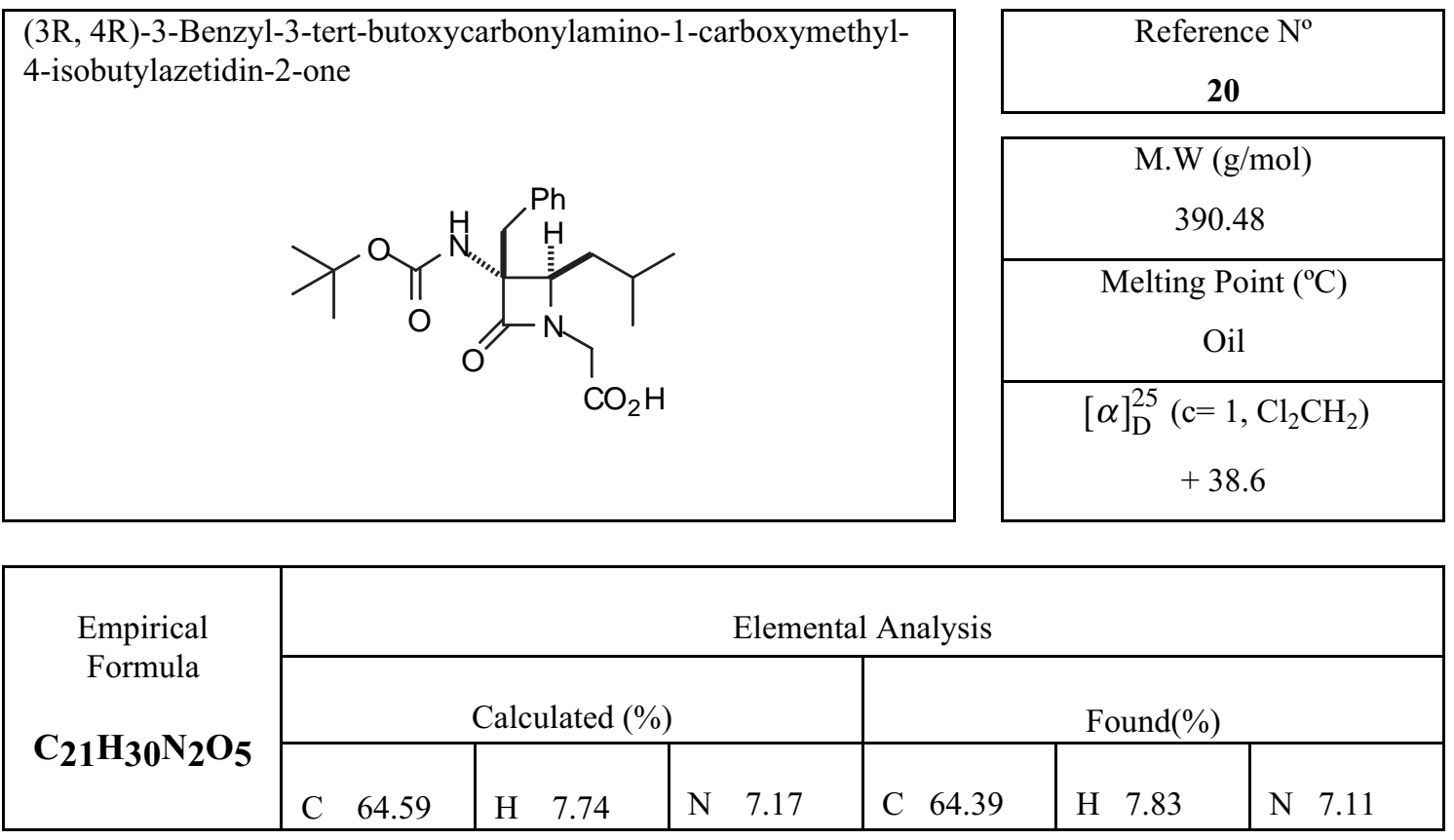

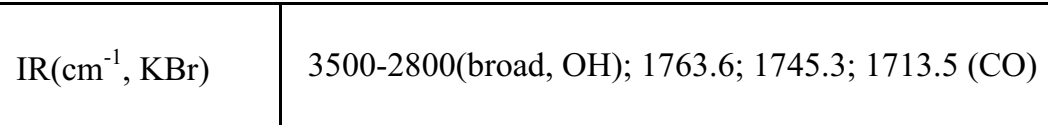

\begin{tabular}{|c|l|}
\hline HPLC-MS & -MS: 389.3 \\
Ion Source Type: & $-M S 2(389.3): 315.1$ \\
ESI* $^{*}$ & $-M S 3(315.1): 272.0,228.0$ \\
& $-M S 4(228.0): 213.0,171.0,150.0,131.0$ \\
\hline
\end{tabular}

\begin{tabular}{|c|c|}
\hline${ }^{1} \mathrm{H}-\mathrm{NMR}(\delta, \mathrm{ppm}) \mathrm{CDCl}_{3}$ & \\
\hline $\begin{array}{l}\text { 7.34-7.29 (m, 5H, Ar) } \\
4.94(\mathrm{~s}, 1 \mathrm{H}, \mathrm{N} \underline{\mathrm{HBoc}}) \\
4.32\left(\mathrm{~d}, 1 \mathrm{H}, \underline{\mathrm{NCH}} \underline{\mathrm{HCO}}{ }_{2} \mathrm{H}\right) \\
4.13\left(\mathrm{~m}, 1 \mathrm{H},(\mathrm{CO}) \mathrm{NC}^{\mathrm{H}}\right) \\
3.91\left(\mathrm{~d}, 1 \mathrm{H}, \mathrm{NCH} \underline{\mathrm{HCO}}{ }_{2} \mathrm{H}\right) \\
3.16\left(\mathrm{~s}, 2 \mathrm{H}, \mathrm{CH}_{2} \mathrm{Ph}\right) \\
1.73-1.66\left(\mathrm{~m}, 2 \mathrm{H},-\mathrm{CH}_{2} \mathrm{CH}\left(\mathrm{CH}_{3}\right)_{2}\right) \\
1.48-1.32\left(\mathrm{~m}, 2 \mathrm{H},-\underline{\mathrm{H}}_{2} \underline{\mathrm{C}}\left(\mathrm{CH}_{3}\right)_{2}\right) \\
1.43\left(\mathrm{~s}, 9 \mathrm{H},(\mathrm{CO}) \mathrm{OC}\left(\underline{\mathrm{CH}}_{3}\right)_{3}\right) \\
1.01\left(\mathrm{~d}, 3 \mathrm{H},-\mathrm{CH}_{2} \mathrm{CH}\left(\mathrm{CH}_{3}\right)\right. \\
1.00\left(\mathrm{~d}, 3 \mathrm{H},-\mathrm{CH}_{2} \mathrm{CH}\left(\underline{\mathrm{CH}}_{3}\right)\right.\end{array}$ & $\begin{array}{l}\mathrm{J}=17.1 \mathrm{~Hz} \\
\mathrm{~J}=17.2 \mathrm{~Hz}\end{array}$ \\
\hline
\end{tabular}

\begin{tabular}{|cc|}
\hline${ }^{13} \mathrm{C}-\mathrm{NMR}(\delta, \mathrm{ppm}) \mathrm{CDCl}_{3}$ \\
\hline 170.7 & 35.3 \\
169.1 & 28.2 \\
154.8 & 26.0 \\
135.0 & 23.4 \\
130.3 & 22.0 \\
128.3 & \\
126.9 & \\
80.3 & \\
69.0 & \\
63.9 & \\
42.3 & \\
37.9 & \\
& \\
\end{tabular}

Comments: ${ }^{*} \mathrm{MeCN} / \mathrm{NH}_{3}$, negative polarity 
(3S, 4R)-3-Benzyl-3-benzylideneamino-4-isobutyl-1-

[bis(trimethylsilyl)methyl]azetidin-2-one
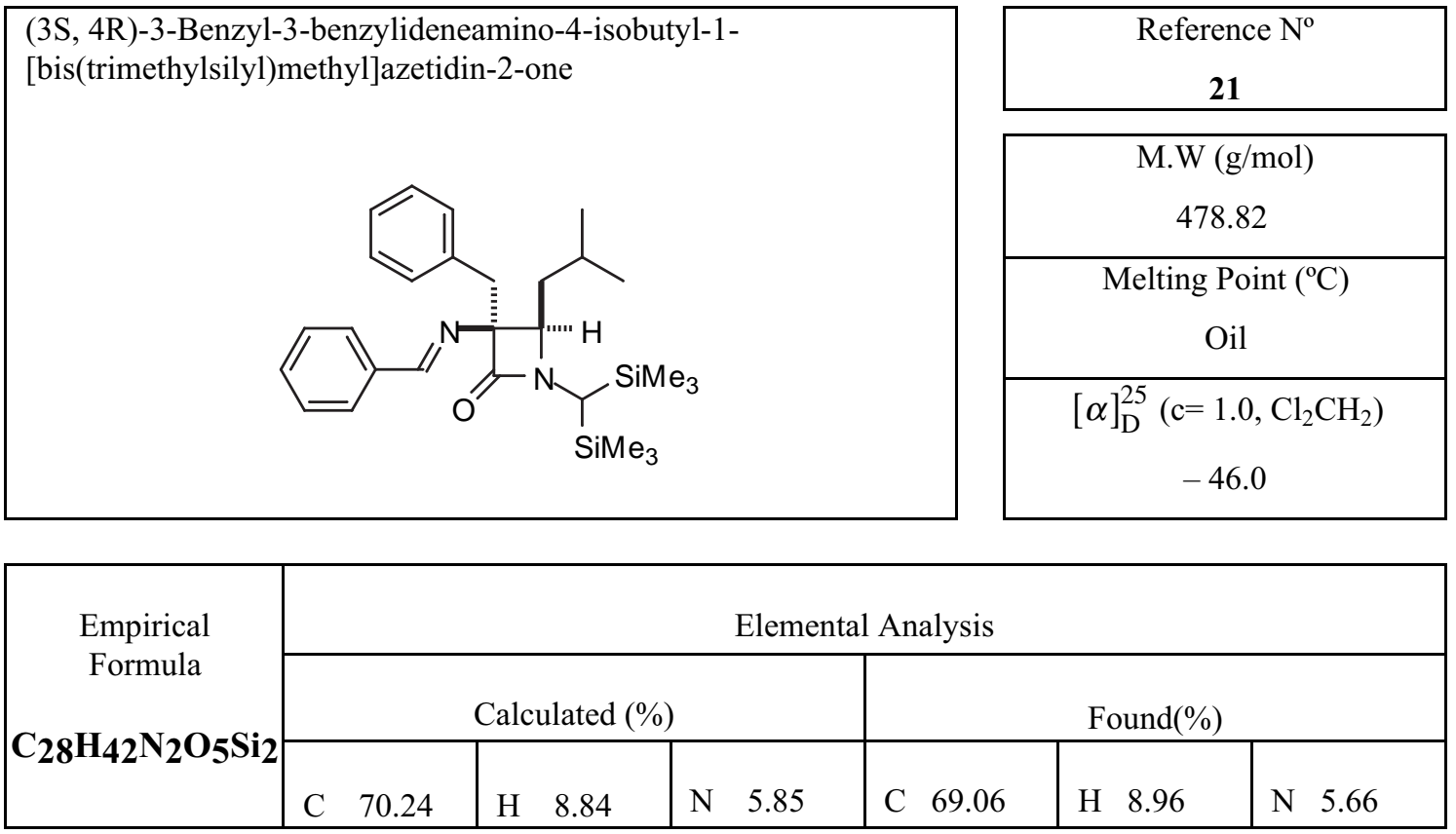

\begin{tabular}{|l|l}
\hline $\operatorname{IR}\left(\mathrm{cm}^{-1}, \mathrm{KBr}\right)$ & $1715.3(\mathrm{C}=\mathrm{O}), 1250.0,848.2(\mathrm{C}-\mathrm{Si})$
\end{tabular}

\begin{tabular}{|c|l|}
\hline HPLC-MS & + MS: 479.4 \\
$\begin{array}{c}\text { Ion Source Type: } \\
\text { ESI* }\end{array}$ & + MS2 (479.4): 463.2, 358.2, 278.2, 244.2, 208.1 \\
& + MS3 (278.2): 220.1, 187.1, 105.2, 91.2 \\
\hline
\end{tabular}

\begin{tabular}{|ll|}
\hline${ }^{1} \mathrm{H}-\mathrm{NMR}(\delta, \mathrm{ppm}) \mathrm{CDCl}_{3}$ & \\
\hline & \\
$8.71(1 \mathrm{H}, \mathrm{NC} \underline{\mathrm{HPh}})$ & \\
$7.8-7.3(\mathrm{~m}, 10 \mathrm{H}$, arom $)$ & \\
$3.76-3.71(\mathrm{dd}, 1 \mathrm{H}, \mathrm{NC} \underline{\mathrm{HiBu})}$ & $\mathrm{J}_{1}=3.3 \mathrm{~Hz}$, \\
& $\mathrm{J}_{2}=10.3 \mathrm{~Hz}$ \\
$3.15\left(\mathrm{dd}, 2 \mathrm{H}, \mathrm{CH}_{2} \mathrm{Ph}\right)$ & $\mathrm{J}_{1}=4.4 \mathrm{~Hz}$, \\
& $\mathrm{J}_{2}=18.1 \mathrm{~Hz}$ \\
$2.08(\mathrm{~s}, 1 \mathrm{H}, \mathrm{C} \underline{\mathrm{HSi}})$ & \\
$1.74\left(\mathrm{~m}, 1 \mathrm{H}, \underline{\mathrm{H}}_{3} \mathrm{CHCH}\right.$ & \\
$1.40-1.24\left(\mathrm{~m}, 2 \mathrm{H}_{3}, \mathrm{CH}_{2} \mathrm{CH}\left(\mathrm{CH}_{3}\right)_{2}\right)$ & \\
$0.95\left(\mathrm{~d}, 3 \mathrm{H}, \mathrm{CH}_{3}\right)$ & $\mathrm{J}=6.6 \mathrm{~Hz}$ \\
$0.89\left(\mathrm{~d}, 3 \mathrm{H}, \underline{\mathrm{C}}_{3}\right)$ & $\mathrm{J}=6.6 \mathrm{~Hz}$ \\
$0.13\left(\mathrm{~s}, 9 \mathrm{H},\left(\underline{\mathrm{CH}}_{3}\right)_{3} \mathrm{Si}\right)$ & \\
$0.05\left(\mathrm{~s}, 9 \mathrm{H},\left(\mathrm{CH}_{3}\right)_{3} \mathrm{Si}\right)$ & \\
& \\
& \\
\hline
\end{tabular}

\begin{tabular}{|cr|}
\hline${ }^{13} \mathrm{C}-\mathrm{NMR}(\delta, \mathrm{ppm}) \mathrm{CDCl}_{3}$ \\
\hline & \\
168.3 & 35.9 \\
161.3 & 24.8 \\
136.7 & 21.9 \\
136.1 & 0.11 \\
130.9 & -0.18 \\
130.7 & \\
130.4 & \\
130.0 & \\
129.7 & \\
128.5 & \\
126.7 & \\
62.6 & \\
42.6 & \\
36.9 & \\
\end{tabular}

Comments: ${ }^{*} \mathrm{MeCN} / \mathrm{NH}_{3}$, positive polarity 
(3S,4R)-3-Benzyl-3-tert-butoxycarbonylamino-4-(2-methylpropyl)-azetidin-2-one
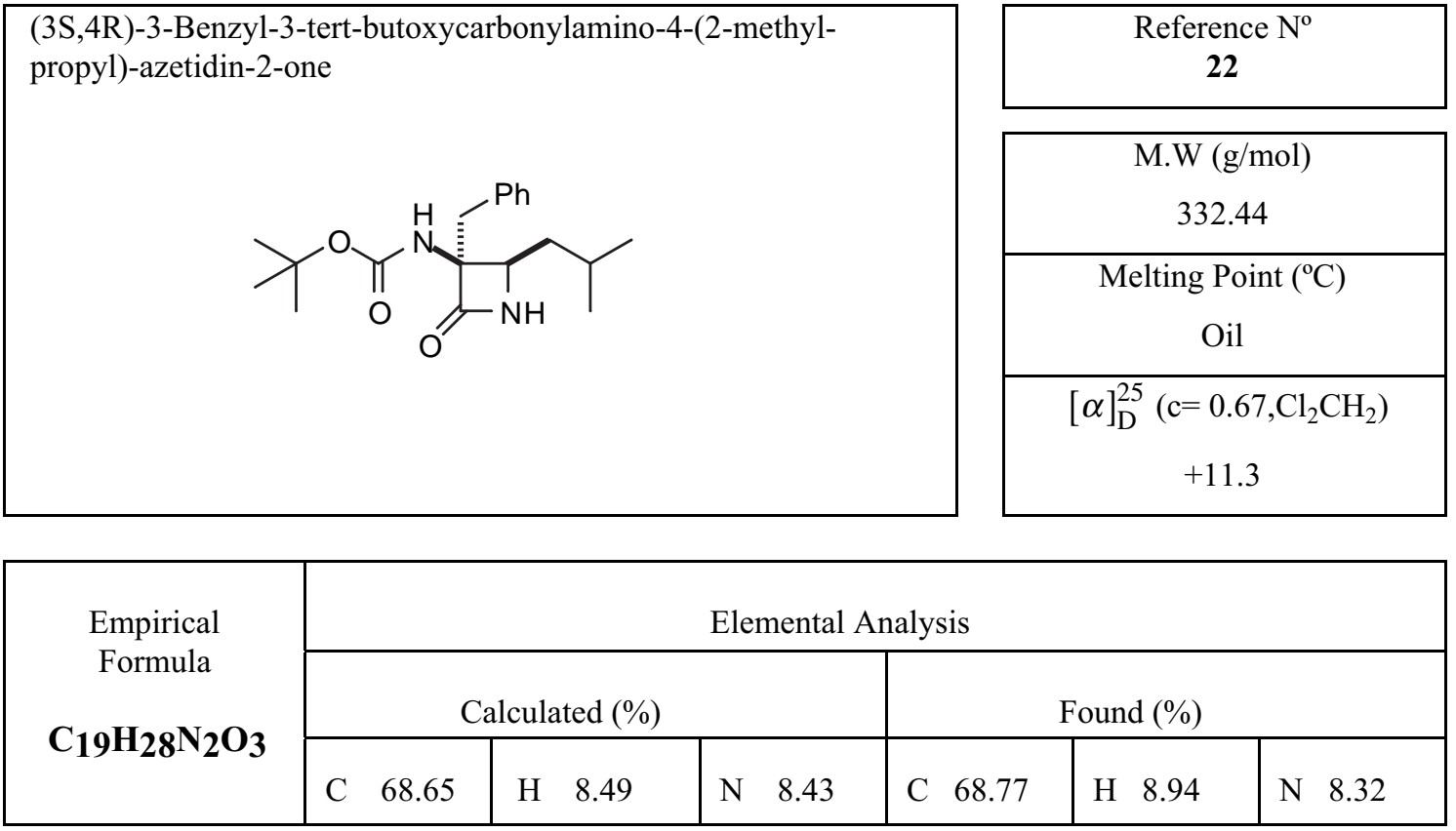

\begin{tabular}{|l|l|}
\hline $\mathrm{IR}\left(\mathrm{cm}^{-1}, \mathrm{KBr}\right)$ & $3171.0(\mathrm{~N}-\mathrm{H}) ; 2904.5 ; 2896.0 ; 1700.8 ; 1686.0(\mathrm{C}=\mathrm{O})$
\end{tabular}

MS m/z

91(24.8); 129(41.7); 146(27.2); 177(100); 214(11.6); 233(49.9); 276(9.4); $289(3.3) ; 333$

\begin{tabular}{|ll|}
\hline${ }^{1} \mathrm{H}-\mathrm{NMR}(\delta, \mathrm{ppm}) \mathrm{CDCl}_{3}$ & \\
\hline & \\
$7.28-7.20(\mathrm{~m}, 5 \mathrm{H}, \mathrm{Ar})$ & \\
$5.83\left(\mathrm{~s}, 1 \mathrm{H}, \mathrm{N} \underline{\mathrm{HC}}\left({ }^{\mathrm{I}} \mathrm{Bu}\right)\right)$ & \\
$4.73(\mathrm{~s}, 1 \mathrm{H}, \mathrm{N} \underline{\mathrm{H}} \mathrm{Boc})$ & \\
$3.69-3.64\left(\mathrm{~m}, 1 \mathrm{H}, \mathrm{HNCH}\left({ }^{\mathrm{i}} \mathrm{Bu}\right)\right.$ & \\
$3.33\left(\mathrm{~d}, 1 \mathrm{H}, \mathrm{CH}_{2} \mathrm{Ph}\right)$ & $\mathrm{J}=13.9 \mathrm{~Hz}$ \\
$3.17\left(\mathrm{~d}, 1 \mathrm{H}, \underline{\mathrm{H}}_{2} \mathrm{Ph}\right)$ & $\mathrm{J}=13.7 \mathrm{~Hz}$ \\
$1.54-1.39\left(\mathrm{~m}, 3 \mathrm{H}, \mathrm{C} \underline{\mathrm{H}}_{2} \mathrm{C} \underline{\mathrm{H}}_{(}\left(\mathrm{CH}_{3}\right)_{2}\right)$ & \\
$1.36\left(\mathrm{~s}, 9 \mathrm{H},(\mathrm{OC}) \mathrm{C}\left(\underline{\mathrm{CH}}_{3}\right)_{3}\right)$ & \\
$0.84\left(\mathrm{~d}, 3 \mathrm{H}, \mathrm{CH}_{2} \mathrm{CH}\left(\underline{\mathrm{CH}}_{3}\right)_{2}\right)$ & $\mathrm{J}=7.3 \mathrm{~Hz}$ \\
$0.82\left(\mathrm{~d}, 3 \mathrm{H}, \mathrm{CH}_{2} \mathrm{CH}\left(\underline{\mathrm{C}}_{3}\right)_{2}\right)$ & $\mathrm{J}=6.3 \mathrm{~Hz}$ \\
& \\
& \\
& \\
\end{tabular}

\begin{tabular}{|c|}
\hline${ }^{13} \mathrm{C}-\mathrm{NMR}(\delta, \mathrm{ppm}) \mathrm{CDCl}_{3}$ \\
\hline 168.5 \\
154.4 \\
135.2 \\
130.2 \\
128.5 \\
127.2 \\
80.1 \\
70.8 \\
58.6 \\
39.8 \\
38.3 \\
28.2 \\
25.7 \\
23.3 \\
22.1 \\
\end{tabular}

Comments: 
(3S, 4R)-3-Benzyl-3-tert-butoxycarbonylamino-1-carboxymethyl-4isobutylazetidin-2-one
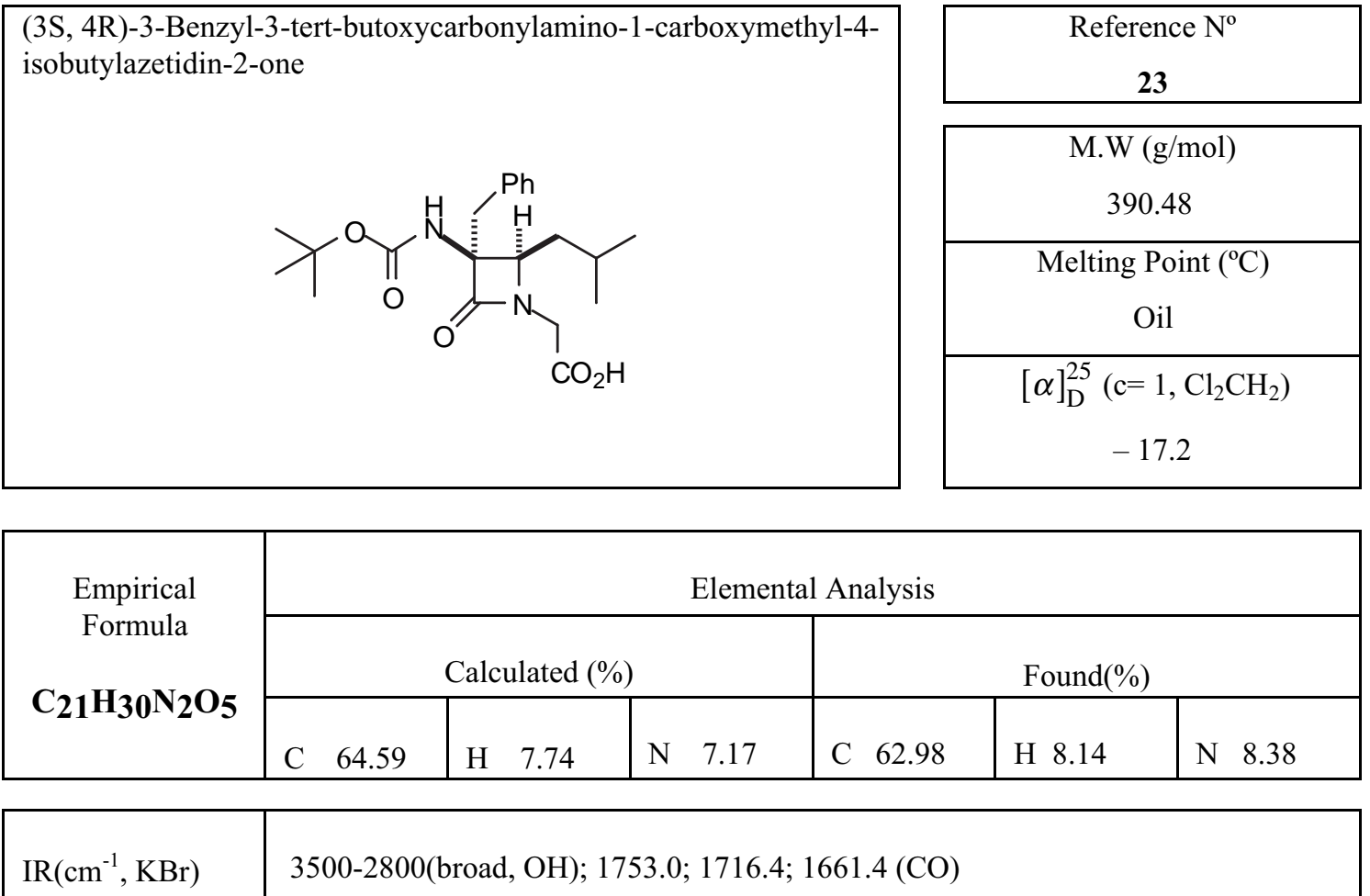

\begin{tabular}{|c|l|}
\hline HPLC-MS & - MS: 389.3 \\
Ion Source Type: & $-M S 2(389.2): 315.1$ \\
ESI* & $-M S 3(315.1): 228.0,150.0$ \\
\hline
\end{tabular}

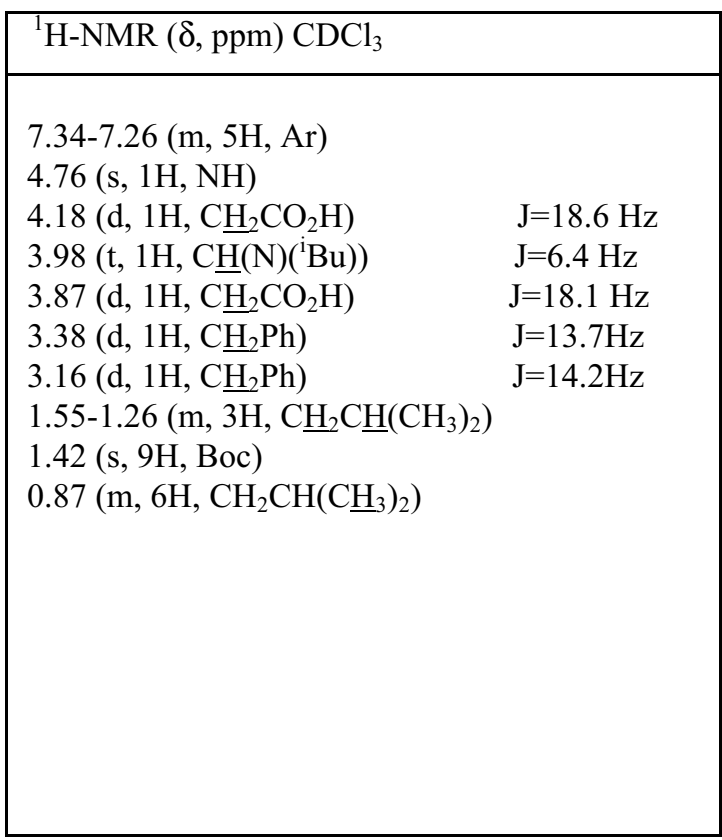

\begin{tabular}{|c|}
\hline${ }^{13} \mathrm{C}-\mathrm{NMR}(\delta, \mathrm{ppm}) \mathrm{CDCl}_{3}$ \\
\hline 170.9 \\
169.0 \\
154.7 \\
134.8 \\
130.0 \\
128.8 \\
127.5 \\
80.7 \\
70.5 \\
63.3 \\
42.0 \\
40.1 \\
36.8 \\
29.7 \\
28.2 \\
25.7 \\
23.0 \\
22.6 \\
\hline
\end{tabular}

Comments: * $\mathrm{MeCN} / \mathrm{NH}_{3}$, negative polarity 
(3R/S,4R)-3-Allyl-1-[bis(trimethylsilyl)methyl]-4-isobutyl-3-

[(4S)-4-phenyl-2-oxo-oxazolidin-3-yl]-azetidin-2-one
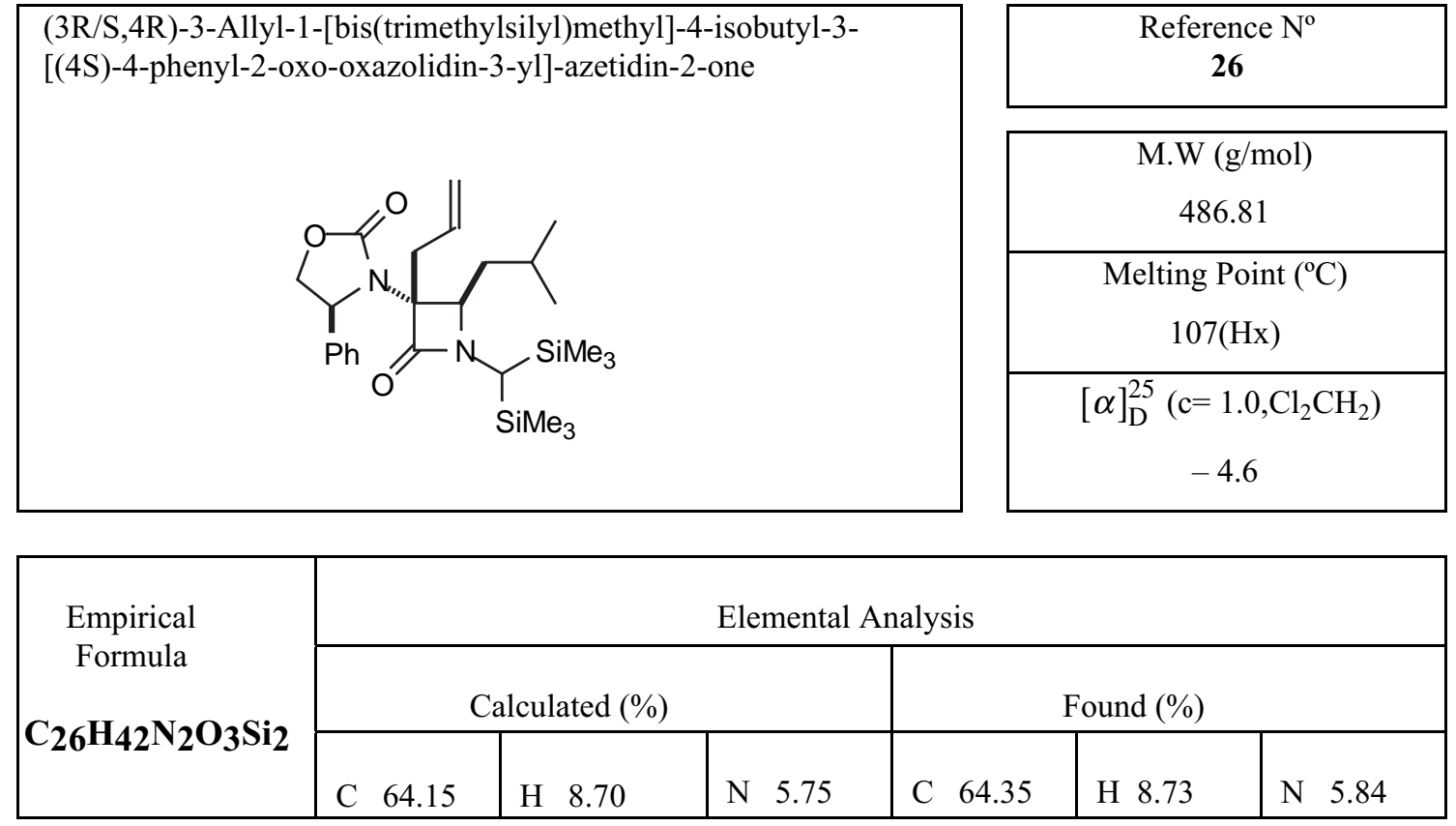

\begin{tabular}{|l|l}
\hline $\mathrm{IR}\left(\mathrm{cm}^{-1}, \mathrm{KBr}\right)$ & $2948.5 ; 2896.5 ; 1742.2$
\end{tabular}

MS m/z

73(44); 91(17); 104(67); 105(25); 198(16); 242(100); 243(20); 285(27); $471(2.3)$

\begin{tabular}{|c|c|}
\hline${ }^{1} \mathrm{H}-\mathrm{NMR}(\delta, \mathrm{ppm}) \mathrm{CDCl}_{3}$ & \\
\hline 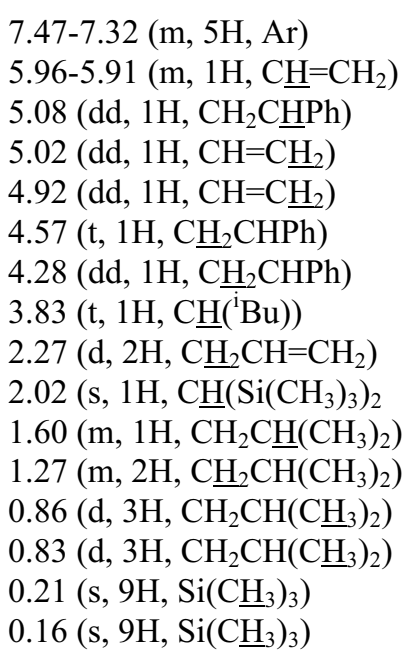 & $\begin{array}{l}\mathrm{J}=1.8,8.1 \mathrm{~Hz} \\
\mathrm{~J}=1.5,10.3 \mathrm{~Hz} \\
\mathrm{~J}=1.6,17.1 \mathrm{~Hz} \\
\mathrm{~J}=8.4 \mathrm{~Hz} \\
\mathrm{~J}=1.8,8.8 \mathrm{~Hz} \\
\mathrm{~J}=6.2 \mathrm{~Hz} \\
\mathrm{~J}=6.6 \mathrm{~Hz}\end{array}$ \\
\hline
\end{tabular}

\begin{tabular}{|cc|}
\hline${ }^{13} \mathrm{C}-\mathrm{NMR}(\delta, \mathrm{ppm}) \mathrm{CDCl}_{3}$ \\
\hline & \\
165.1 & 22.4 \\
156.8 & 0.07 \\
140.8 & \\
132.8 & \\
129.2 & \\
128.8 & \\
127.2 & \\
118.1 & \\
70.9 & \\
69.8 & \\
64.3 & \\
58.8 & \\
37.3 & \\
34.6 & \\
25.2 & \\
23.3 & \\
\end{tabular}

Comments: 
(3R,4R)-1-[Bis(trimethylsilyl)methyl]-3-(4-bromobenzyl)-4-

ethyl-3-[(4S)-4-phenyl-2-oxo-oxazolidin-3-yl]-azetidin-2-one
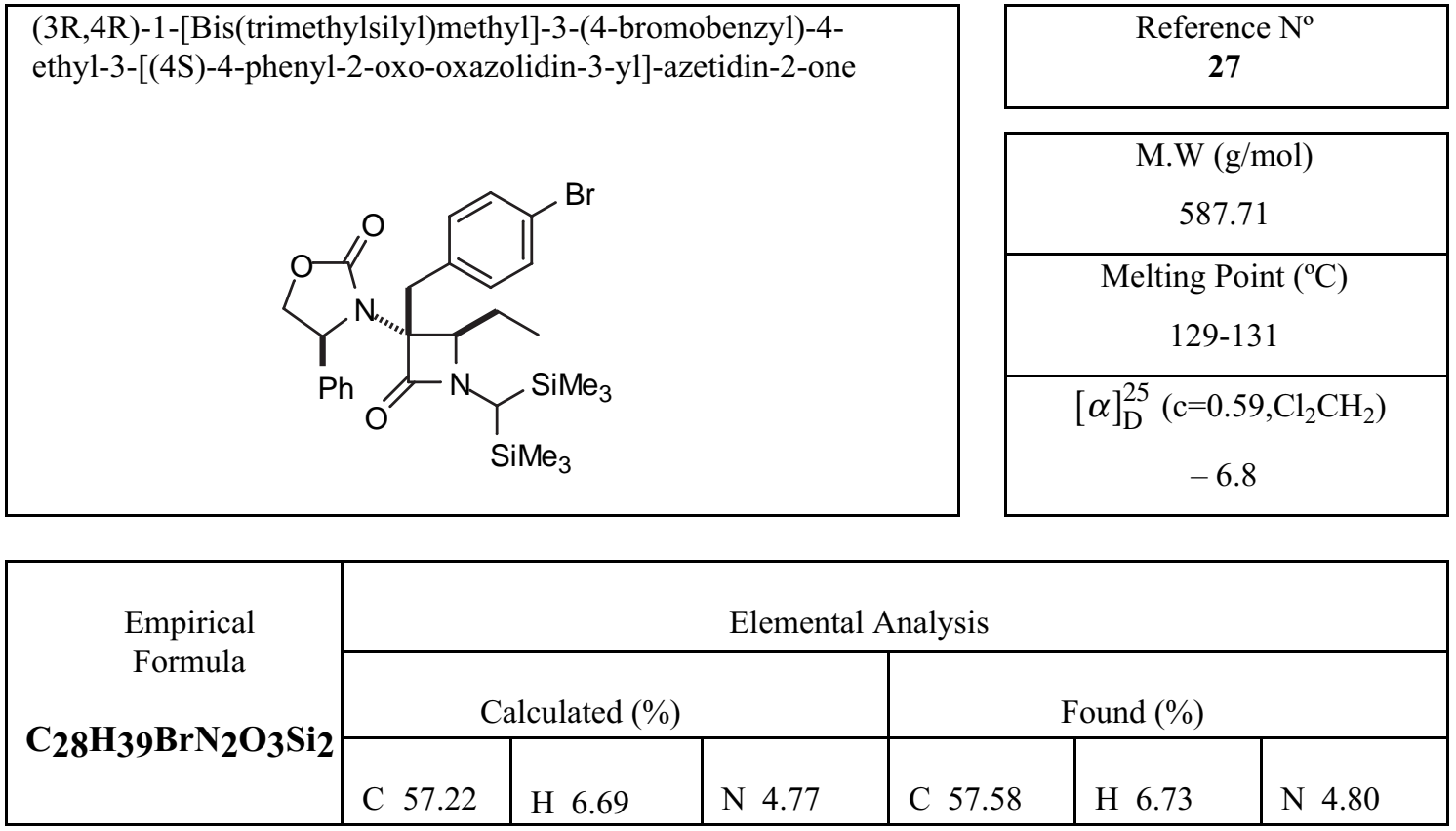

\begin{tabular}{l|l}
\hline $\operatorname{IR}\left(\mathrm{cm}^{-1}, \mathrm{KBr}\right)$ & $2882.5 ; 1729.0(\mathrm{C}=\mathrm{O})$
\end{tabular}

MS m/z

73(33.3); 104(100); 216(58.7); 385(52.3); 387(49.4); 572(3.2); 588(1.4)

\begin{tabular}{|c|}
\hline${ }^{1} \mathrm{H}-\mathrm{NMR}(\delta, \mathrm{ppm}) \mathrm{CDCl}_{3}$ \\
\hline 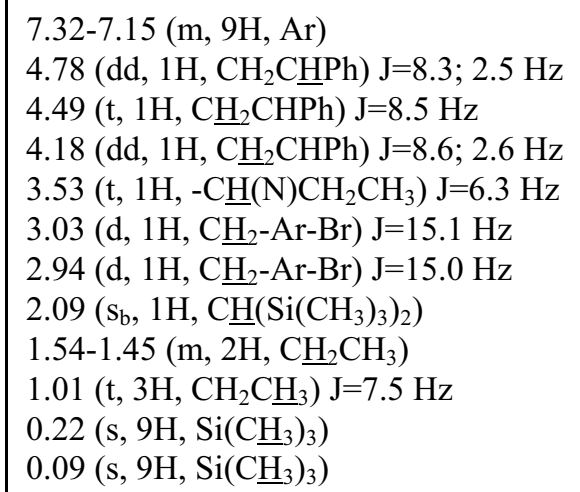 \\
\hline
\end{tabular}

\begin{tabular}{|cc|}
\hline${ }^{13} \mathrm{C}-\mathrm{NMR}(\delta, \mathrm{ppm}) \mathrm{CDCl}_{3}$ \\
\hline 164.4 & 0.20 \\
157.2 & 0.02 \\
140.0 & \\
134.6 & \\
132.1 & \\
131.2 & \\
128.9 & \\
128.5 & \\
127.0 & \\
120.8 & \\
69.7 & \\
59.8 & \\
53.4 & \\
37.7 & \\
35.1 & \\
21.8 & \\
12.1 & \\
\hline
\end{tabular}

Comments: 
(3R)-1-[Bis(trimethylsilyl)methyl]-3-[2-methyl-2-propenyl]-4phenyl-3-[(4S)-4-phenyl-2-oxo-oxazolidin-3-yl]-azetidin-2-one
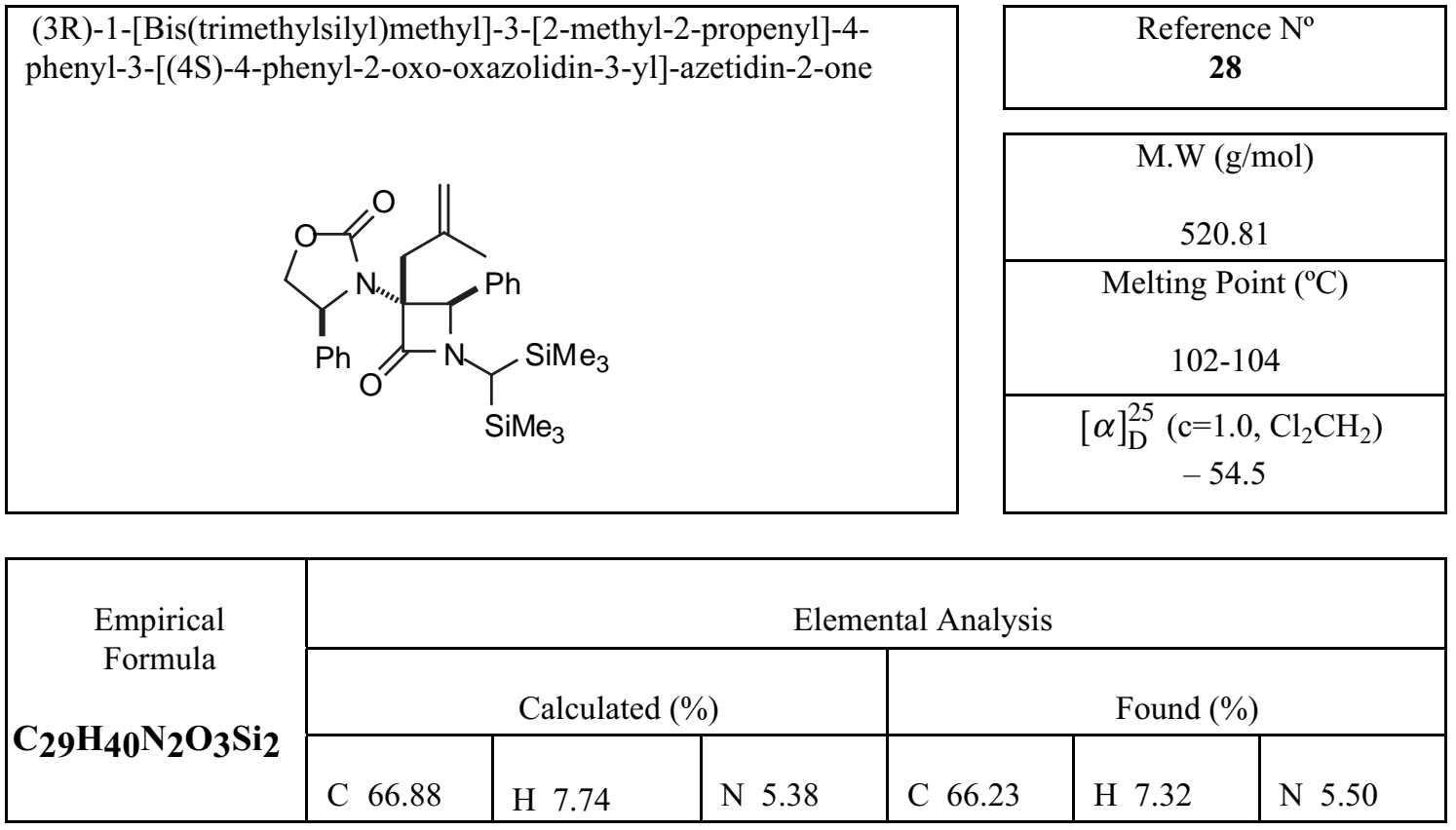

\begin{tabular}{l|l}
\hline $\mathrm{IR}\left(\mathrm{cm}^{-1}, \mathrm{KBr}\right)$ & $2941.0 ; 2892.5 ; 1747.2$
\end{tabular}

MS m/z

73(100); 104(61.3); 143(78.9); 156(69.6); 257(36.2); 319(35.3); 343(42.1); 358(22.8); 506(7.6); 521(2.9)

\begin{tabular}{|c|}
\hline${ }^{1} \mathrm{H}-\mathrm{NMR}(\delta, \mathrm{ppm}) \mathrm{CDCl}_{3}$ \\
\hline $\begin{array}{l}7.40(\mathrm{~s}, 5 \mathrm{H}, \mathrm{ArH}) \\
7.24-7.20(\mathrm{~m}, 3 \mathrm{H}, \mathrm{ArH}) \\
6.80(\mathrm{~m}, 2 \mathrm{H}, \mathrm{ArH}) \\
4.99\left(\mathrm{~d}, 1 \mathrm{H}, \mathrm{CH}_{2} \mathrm{CHPh}\right) \\
4.97\left(\mathrm{~s}, 1 \mathrm{H}, \mathrm{C}=\mathrm{CH}_{2}\right) \\
4.93\left(\mathrm{~s}, 1 \mathrm{H},(\mathrm{C}) \underline{\mathrm{CH} h}^{\mathrm{H} P h}\right. \\
4.73\left(\mathrm{~s}, 1 \mathrm{H}, \mathrm{C}=\mathrm{CH}_{2}\right) \\
4.60\left(\mathrm{t}, 1 \mathrm{H}, \mathrm{C} \underline{\mathrm{H}}_{2} \mathrm{CHPh}\right) \mathrm{J}=8.3 \mathrm{~Hz} \\
4.30\left(\mathrm{~d}, 1 \mathrm{H}, \underline{\mathrm{H}}_{2} \mathrm{CHPh}\right) \mathrm{J}=8.8 \mathrm{~Hz} \\
2.10\left(\mathrm{~s}, 1 \mathrm{H}, \underline{\mathrm{CHSi}}^{\mathrm{H}}\right) \\
2.08\left(\mathrm{~d}, 1 \mathrm{H}, \mathrm{CH}_{2} \mathrm{C}\left(\mathrm{CH}_{3}\right)=\mathrm{CH}_{2}\right) \\
1.97\left(\mathrm{~d}, 1 \mathrm{H}, \mathrm{CH}_{2} \mathrm{C}\left(\mathrm{CH}_{3}\right)=\mathrm{CH}_{2}\right) \mathrm{J}=17.2 \mathrm{~Hz} \\
1.44(\mathrm{~s}, 3 \mathrm{H}, \mathrm{CH})_{3} \\
0.29\left(\mathrm{~s}, 9 \mathrm{H}, \mathrm{Si}\left(\underline{\mathrm{CH}}_{3}\right)_{3}\right) \\
0.19\left(\mathrm{~s}, 9 \mathrm{H}, \mathrm{Si}\left(\underline{\mathrm{CH}}_{3}\right)_{3}\right)\end{array}$ \\
\hline
\end{tabular}

\begin{tabular}{|cc|}
\hline${ }^{13} \mathrm{C}-\mathrm{NMR}(\delta, \mathrm{ppm}) \mathrm{CDCl}_{3}$ \\
\hline 165.9 & 37.9 \\
156.7 & 36.4 \\
141.2 & 23.8 \\
139.2 & 0.75 \\
133.2 & 0.33 \\
129.3 & \\
128.9 & \\
128.3 & \\
128.2 & \\
128.1 & \\
127.4 & \\
114.7 & \\
72.6 & \\
71.0 & \\
69.7 & \\
59.2 & \\
53.4 & \\
\hline
\end{tabular}

Comments: 
(3R,4R)-1-[Bis(trimethylsilyl)methyl]-3-[4-methyl-(phenyl)-methyl]

-4-phenyl-3-[(4S)-4-phenyl-2-oxo-oxazolidin-3-yl]-azetidin-2-one

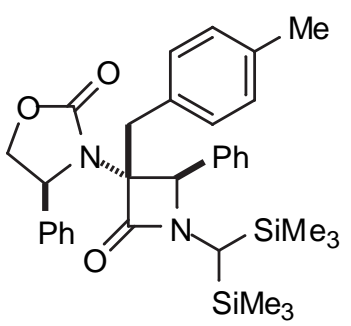

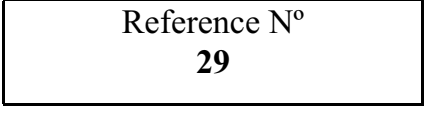

\begin{tabular}{|c|}
\hline M.W $(\mathrm{g} / \mathrm{mol})$ \\
520.81 \\
\hline Melting Point $\left({ }^{\circ} \mathrm{C}\right)$ \\
$132-134$ \\
\hline$[\alpha]_{\mathrm{D}}^{25}\left(\mathrm{c}=1.0, \mathrm{Cl}_{2} \mathrm{CH}_{2}\right)$ \\
-74.7 \\
\hline
\end{tabular}

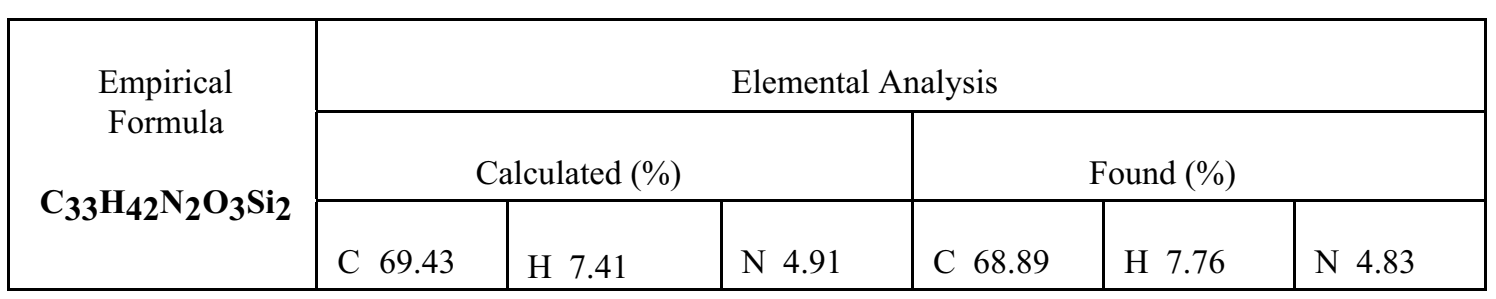

$\operatorname{IR}\left(\mathrm{cm}^{-1}, \mathrm{KBr}\right)$ $2941.0 ; 2895.0 ; 1746.6 ; 1744.9$

MS m/z

\begin{tabular}{|c|}
\hline${ }^{1} \mathrm{H}-\mathrm{NMR}(\delta, \mathrm{ppm}) \mathrm{CDCl}_{3}$ \\
\hline $\begin{array}{l}\text { 7.34-7.19 (m, 8H, Ar) } \\
6.98\left(\mathrm{~d}, 2 \mathrm{H}, \mathrm{Ar}-\mathrm{CH}_{3}\right) \mathrm{J}=8.1 \mathrm{~Hz} \\
6.89\left(\mathrm{~d}, 2 \mathrm{H}, \mathrm{Ar}-\mathrm{CH}_{3}\right) \mathrm{J}=7.7 \mathrm{~Hz} \\
6.83(\mathrm{~d}, 2 \mathrm{H}, \mathrm{Ar}) \mathrm{J}=7.3 \mathrm{~Hz} \\
4.91\left(\mathrm{~s}, 1 \mathrm{H}, \mathrm{PhHC}-\underline{\mathrm{H}}^{-}\right) \\
4.70\left(\mathrm{~d}, 1 \mathrm{H}, \mathrm{CH}_{2} \mathrm{CHPh}\right) \mathrm{J}=8.1 \mathrm{~Hz} \\
4.39\left(\mathrm{t}, 1 \mathrm{H}, \mathrm{CH}_{2} \mathrm{CHPh}\right) \mathrm{J}=8.2 \mathrm{~Hz} \\
4.18\left(\mathrm{~d}, 1 \mathrm{H}, \mathrm{CH}_{2} \mathrm{CHPh}\right) \mathrm{J}=7.0 \mathrm{~Hz} \\
2.86\left(\mathrm{~d}, 1 \mathrm{H}, \mathrm{CH}_{2}-\mathrm{Ar}-\mathrm{CH}_{3}\right) \mathrm{J}=16.1 \mathrm{~Hz} \\
2.47\left(\mathrm{~d}, 1 \mathrm{H}, \mathrm{CH}_{2}-\mathrm{Ar}-\mathrm{CH}_{3}\right) \mathrm{J}=16.1 \mathrm{~Hz} \\
2.26\left(\mathrm{~s}, 3 \mathrm{H}, \mathrm{CH}_{3}\right) \\
\left.2.15\left(\mathrm{~s}, 1 \mathrm{H}, \mathrm{CH}_{(\mathrm{Si}}\left(\mathrm{CH}_{3}\right)_{3}\right)_{2}\right) \\
0.27\left(\mathrm{~s}, 9 \mathrm{H}, \mathrm{Si}\left(\mathrm{CH}_{3}\right)_{3}\right) \\
0.20\left(\mathrm{~s}, 9 \mathrm{H}, \mathrm{Si}\left(\underline{\mathrm{CH}}_{3}\right)_{3}\right)\end{array}$ \\
\hline
\end{tabular}

\begin{tabular}{|cc|}
\hline${ }^{13} \mathrm{C}-\mathrm{NMR}(\delta, \mathrm{ppm}) \mathrm{CDCl}_{3}$ \\
\hline 165.9 & 59.1 \\
157.0 & 36.7 \\
140.8 & 36.3 \\
135.8 & 21.0 \\
133.2 & 0.81 \\
132.2 & 0.37 \\
129.5 & \\
129.3 & \\
128.8 & \\
128.7 & \\
128.4 & \\
128.3 & \\
128.0 & \\
127.1 & \\
73.4 & \\
71.0 & \\
70.0 & \\
\end{tabular}

Comments: 
(3R)-1-[Bis(trimethylsilyl)methyl]-3-isobutyl-3-[(4S)-4-

phenyl-2-oxo-oxazolidin-3-yl]-azetidin-2-one
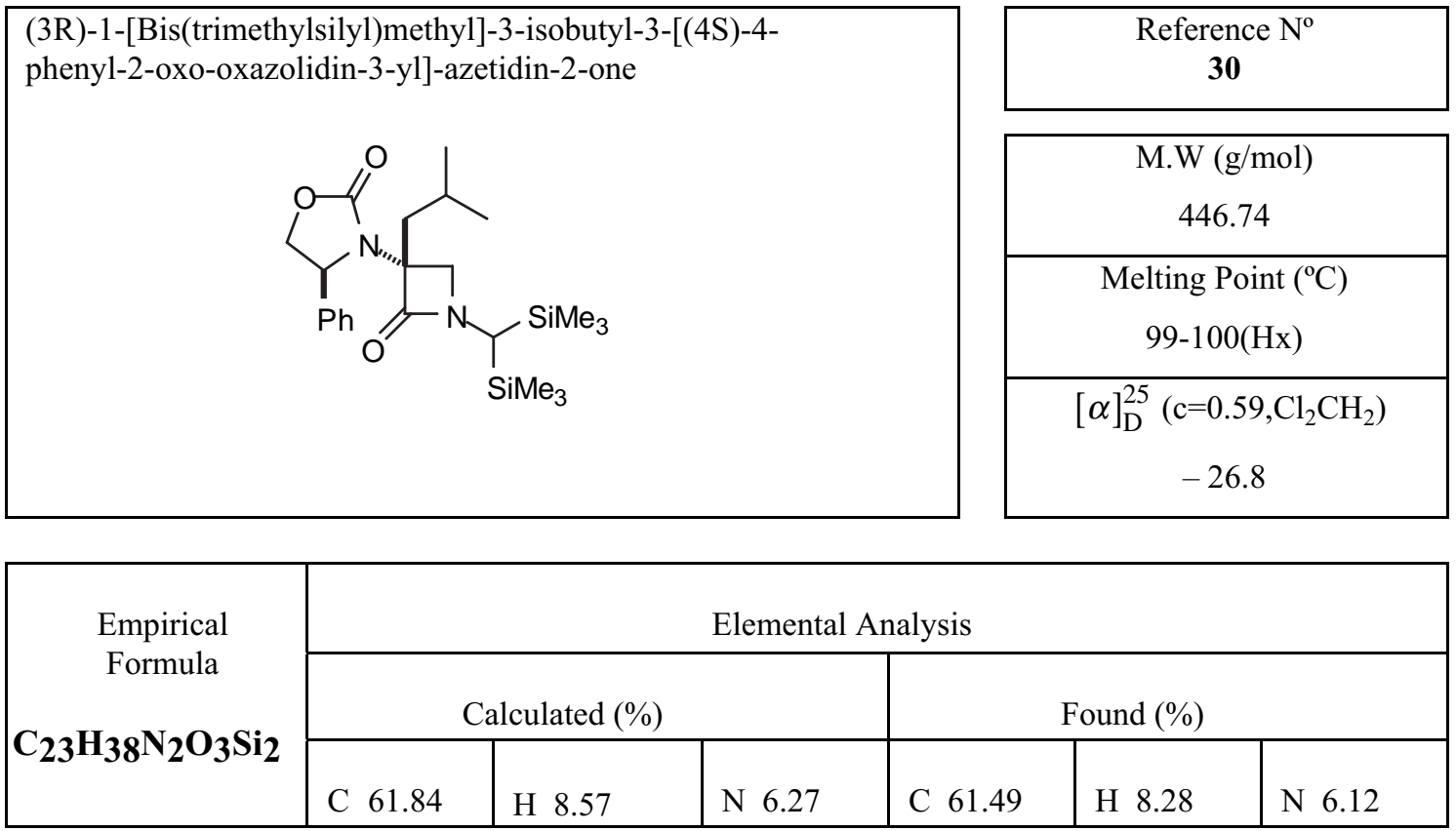

\begin{tabular}{|l|l}
\hline $\mathrm{IR}\left(\mathrm{cm}^{-1}, \mathrm{KBr}\right)$ & $1738.4(\mathrm{C}=\mathrm{O})$
\end{tabular}

$\mathrm{MS} \mathrm{m} / \mathrm{z}$

68(100); 73(100); 104(100); 118(39.1); 204(100); 205(40.9); 246(36.9)

\begin{tabular}{|ll|}
\hline${ }^{1} \mathrm{H}-\mathrm{NMR}(\delta, \mathrm{ppm}) \mathrm{CDCl}_{3}$ & \\
\hline & \\
$7.57(\mathrm{~m}, 2 \mathrm{H}, \mathrm{Ph})$ & \\
$7.38(\mathrm{~m}, 3 \mathrm{H}, \mathrm{Ph})$ & \\
$5.19(\mathrm{dd}, 1 \mathrm{H}, \mathrm{OC} \underline{\mathrm{HHCHPh}})$ & $\mathrm{J}=8.4,2.3 \mathrm{~Hz}$ \\
$4.61(\mathrm{t}, 1 \mathrm{H}, \underline{\mathrm{HCPh}})$ & $\mathrm{J}=8.6 \mathrm{~Hz}$ \\
$4.41(\mathrm{dd}, 1 \mathrm{H}, \mathrm{OCH} \underline{\mathrm{H}} \mathrm{HPh})$ & $\mathrm{J}=8.8,2.3 \mathrm{~Hz}$ \\
$3.89(\mathrm{~d}, 1 \mathrm{H}, \mathrm{HC} \underline{\mathrm{HN}})$ & $\mathrm{J}=6.8 \mathrm{~Hz}$ \\
$3.50(\mathrm{~d}, 1 \mathrm{H}, \underline{\mathrm{H}} \mathrm{HN})$ & $\mathrm{J}=6.8 \mathrm{~Hz}$ \\
$2.70(\mathrm{~s}, 1 \mathrm{H}, \underline{\mathrm{CHSi}})$ & \\
$1.6-1.3(\mathrm{~m}, 3 \mathrm{H}, \mathrm{CH}$ & y CH alkyl $)$ \\
$0.89\left(\mathrm{~d}, 3 \mathrm{H}, \mathrm{CH}_{3}\right)$ & $\mathrm{J}=6.3 \mathrm{~Hz}$ \\
$0.49\left(\mathrm{~d}, 3 \mathrm{H}, \mathrm{CH}_{3}\right)$ & $\mathrm{J}=6.3 \mathrm{~Hz}$ \\
$0.15\left(\mathrm{~s}, 9 \mathrm{H},\left(\mathrm{CH}_{3}\right)_{3} \mathrm{Si}\right)$ & \\
$0.14\left(\mathrm{~s}, 9 \mathrm{H},\left(\mathrm{CH}_{3}\right)_{3} \mathrm{Si}\right)$ & \\
& \\
& \\
& \\
\end{tabular}

\begin{tabular}{|c|}
\hline${ }^{13} \mathrm{C}-\mathrm{NMR}(\delta, \mathrm{ppm}) \mathrm{CDCl}_{3}$ \\
\hline 165.9 \\
140.4 \\
128.8 \\
127.7 \\
71.0 \\
70.7 \\
59.5 \\
54.5 \\
41.9 \\
37.0 \\
24.1 \\
24.0 \\
22.9 \\
-0.2 \\
-0.3 \\
\end{tabular}

Comments: Crystallized from boiling hexane. White solid. 
(3R)-1-[(Aminocarbonyl)methyl]-3-(2-methyl-propyl)-3-[(4S)-

4-phenyl-2-oxo-oxazolidin-3-yl]-azetidin-2-one
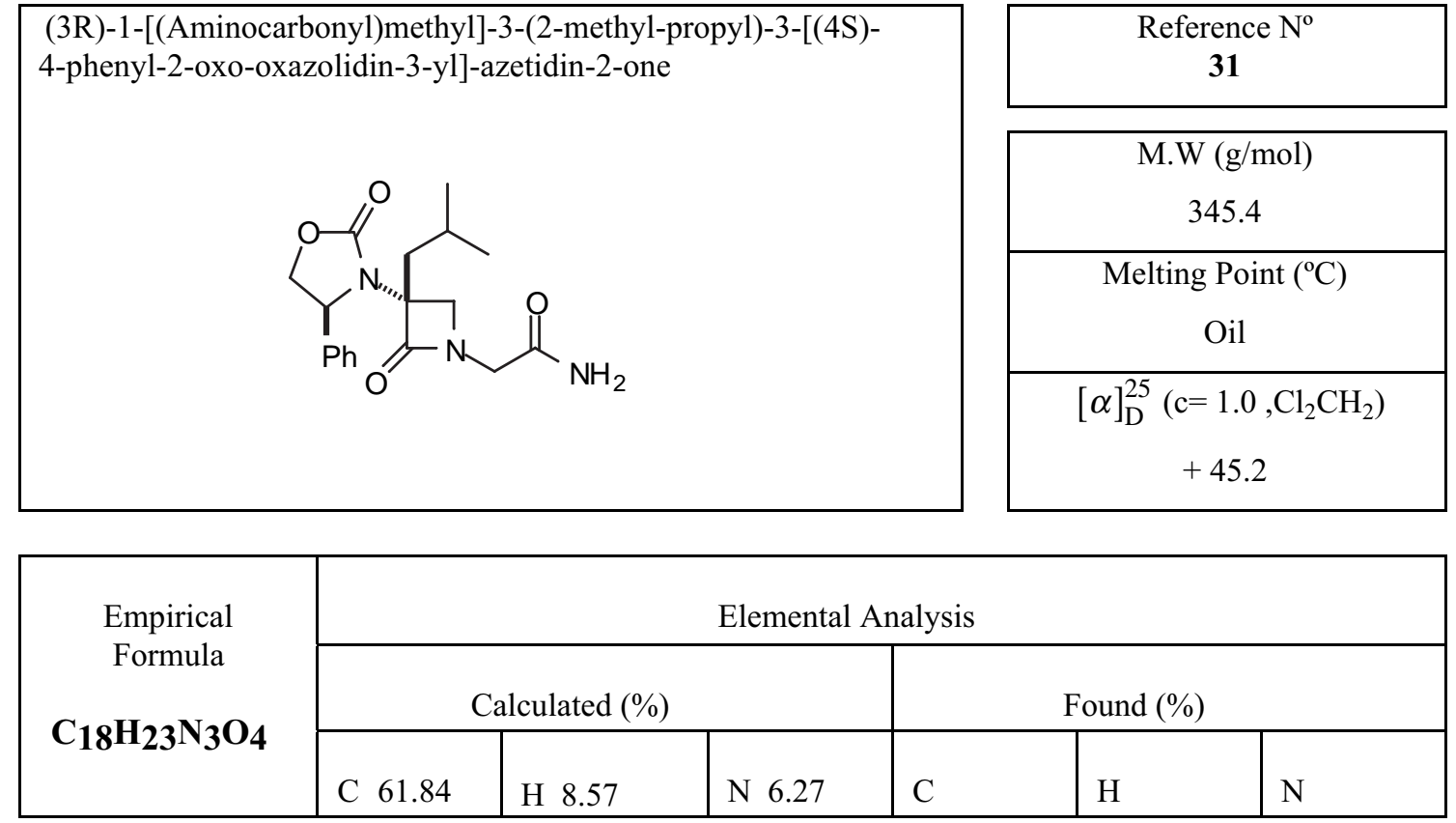

\begin{tabular}{|l|l|}
\hline $\mathrm{IR}\left(\mathrm{cm}^{-1}, \mathrm{film}\right)$ & $3280(\mathrm{NH}), 2920,1717,1669,1604(\mathrm{C}=\mathrm{O})$ \\
\hline
\end{tabular}

MS m/z

68(36); 91(27); 104(82); 118(94); 202(33); 203(100)

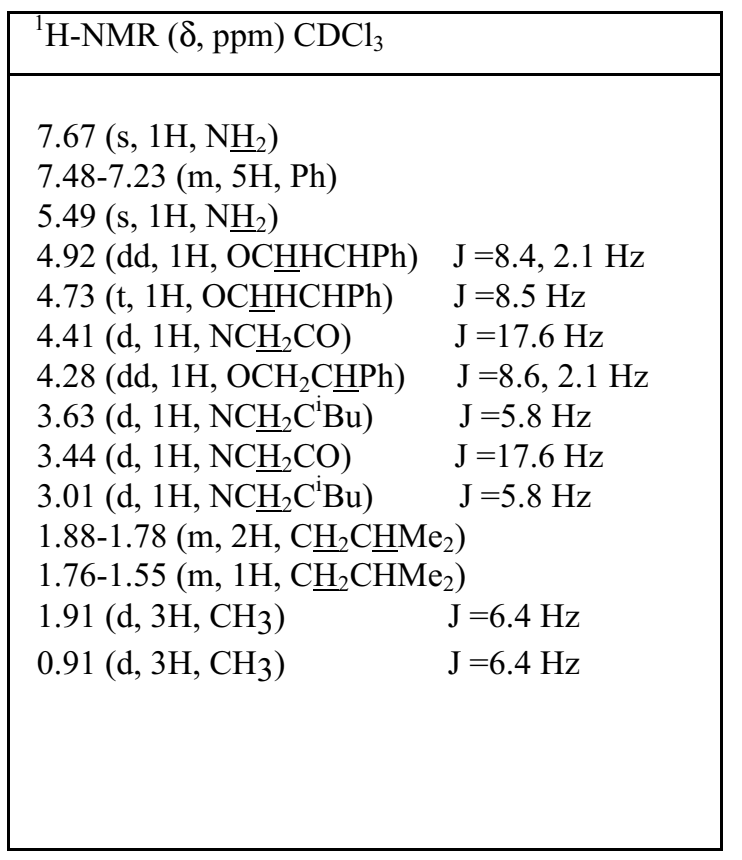

\begin{tabular}{|l|}
\hline${ }^{13} \mathrm{C}-\mathrm{NMR}(\delta, \mathrm{ppm}) \mathrm{CDCl}_{3}$ \\
\hline 169.7 \\
166.6 \\
157.2 \\
139.6 \\
129.4 \\
129.2 \\
126.2 \\
70.7 \\
70.4 \\
59.1 \\
53.4 \\
44.8 \\
40.9 \\
24.3 \\
23.9 \\
23.6
\end{tabular}

Comments: Analytically pure sample by preparative HPLC (EtOAc) 
(3R) 1-Amidomethyl-3-isobutyl-3-[(2S)-1-(tert-butoxycar

bonyl)-pyrrolidin-2-yl-carbonylamino]azetidin-2-one.
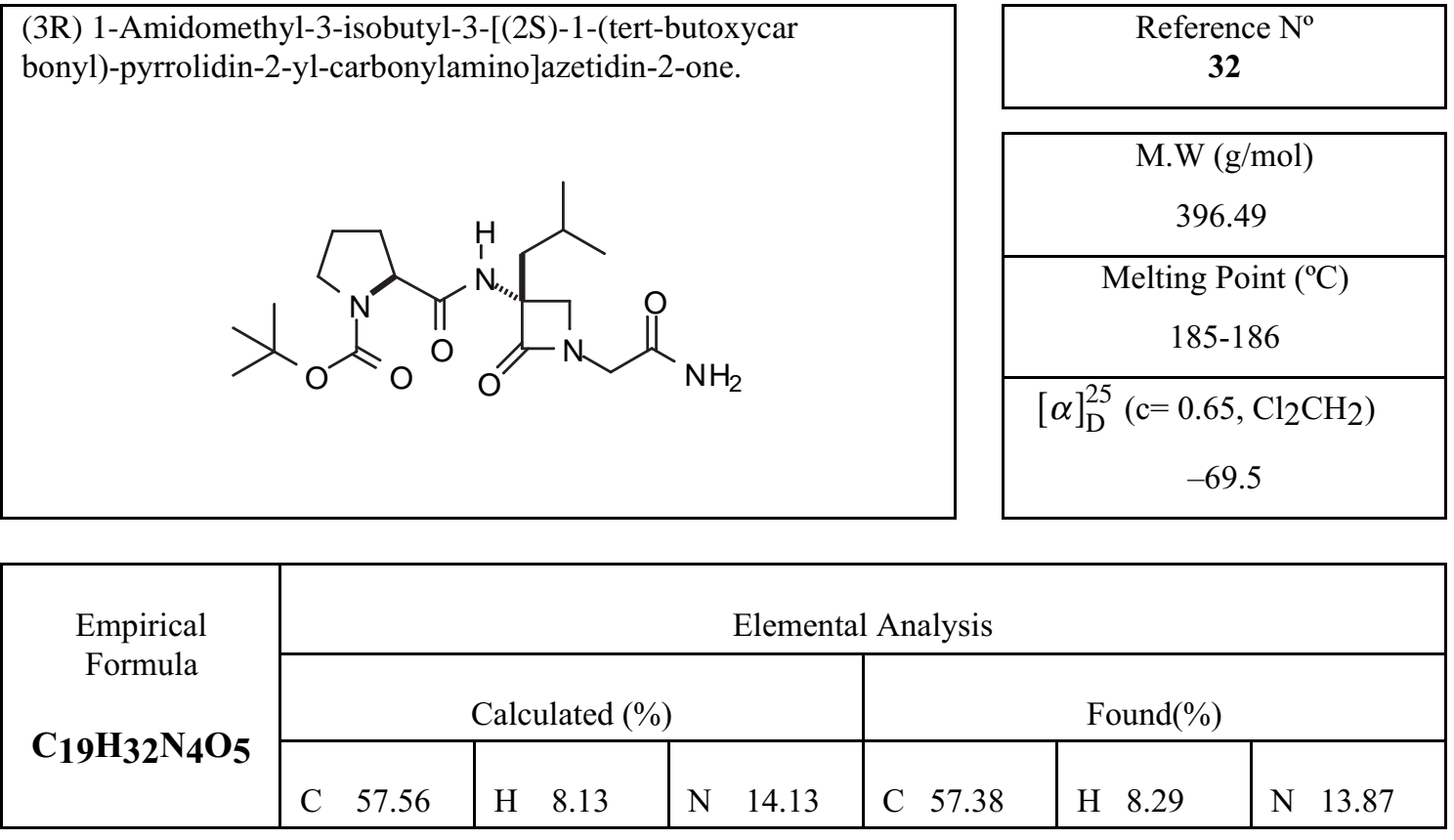

$\operatorname{IR}\left(\mathrm{cm}^{-1}, \mathrm{KBr}\right)$

\begin{tabular}{|c|l|}
\hline MS & - MS: 395.6 \\
$\begin{array}{c}\text { Ion Source Type: } \\
\text { ESI }\end{array}$ & - MS2(395.7): 321.2, 309.4, 139.1 \\
\hline
\end{tabular}

\begin{tabular}{|c|c|}
\hline${ }^{1} \mathrm{H}-\mathrm{NMR}(\delta, \mathrm{ppm}) \mathrm{CDCl}_{3}$ & \\
\hline $\begin{array}{l}8.28\left(\mathrm{~s}, 1 \mathrm{H},-(\mathrm{CO}) \mathrm{NH}_{2}\right) \\
8.02\left(\mathrm{~s}, 1 \mathrm{H},-(\mathrm{CO}) \mathrm{NH}^{-}\right) \\
5.50\left(\mathrm{~s}, 1 \mathrm{H},-(\mathrm{CO}) \mathrm{NH}_{2}\right) \\
4.47\left(\mathrm{~d}, 1 \mathrm{H},-\mathrm{NC} \underline{\mathrm{H}}_{2}(\mathrm{CO})-\right) \\
4.34(\mathrm{~s}, 1 \mathrm{H},-\mathrm{N}(\mathrm{Boc})-\mathrm{CH}(\mathrm{CO})) \\
3.83\left(\mathrm{~d}, 1 \mathrm{H},-\mathrm{NC} \underline{\mathrm{H}}_{2} \mathrm{C}\left({ }^{\mathrm{I}} \mathrm{Bu}\right)\right) \\
3.44\left(\mathrm{~d}, 1 \mathrm{H},-\mathrm{NCH}_{2}(\mathrm{CO})-\right) \\
3.35\left(\mathrm{~d}, 1 \mathrm{H},-\mathrm{NC} \underline{\mathrm{H}}_{2} \mathrm{C}\left({ }^{\mathrm{I}} \mathrm{Bu}\right)\right) \\
3.46-3.34\left(\mathrm{~m}, 2 \mathrm{H},-\mathrm{CH}_{2} \mathrm{CH}_{2} \mathrm{~N}(\mathrm{Boc}\right. \\
\left.1.99-1.70\left(\mathrm{~m}, 3 \mathrm{H}, \mathrm{CH}_{2} \underline{\mathrm{CH}}_{(\mathrm{CH}}\right)_{2}\right) \\
1.48\left(\mathrm{~s}, 9 \mathrm{H}, \mathrm{O}(\mathrm{CO}) \mathrm{C}\left(\mathrm{CH}_{3}\right)_{3}\right) \\
1.03\left(\mathrm{~d}, 3 \mathrm{H}, \mathrm{CH}_{2} \mathrm{CH}\left(\mathrm{CH}_{3}\right)_{2}\right) \\
0.94\left(\mathrm{~d}, 3 \mathrm{H}, \mathrm{CH}_{2} \mathrm{CH}\left(\mathrm{CH}_{3}\right)_{2}\right)\end{array}$ & $\begin{array}{l}\mathrm{J}=17.8 \mathrm{~Hz} \\
\mathrm{~J}=4.9 \mathrm{~Hz} \\
\mathrm{~J}=17.8 \mathrm{~Hz} \\
\mathrm{~J}=5.2 \mathrm{~Hz} \\
-) \\
\\
\mathrm{J}=6.4 \mathrm{~Hz} \\
\mathrm{~J}=5.7 \mathrm{~Hz}\end{array}$ \\
\hline
\end{tabular}

\begin{tabular}{|cc|}
\hline${ }^{13} \mathrm{C}-\mathrm{NMR}(\delta, \mathrm{ppm}) \mathrm{CDCl}_{3}$ \\
\hline & \\
172.2 & 24.5 \\
170.5 & 23.9 \\
168.7 & 22.3 \\
156.5 & \\
80.9 & \\
68.1 & \\
59.3 & \\
53.4 & \\
47.3 & \\
44.9 & \\
42.1 & \\
39.7 & \\
28.3 & \\
26.4 & \\
\end{tabular}

Comments: $* \mathrm{MeCN} / \mathrm{NH}_{3}$, negative polarity 


\section{CRYSTALLOGRAPHIC ANALYSIS OF COMPOUNDS 11, 18 AND 32.}

Crystal data for compound 11: $\mathrm{C}_{25} \mathrm{H}_{37} \mathrm{~N}_{3} \mathrm{O}_{6}, M_{\mathrm{r}}=475.58$, colorless prisms, trigonal, space group $P 4_{3} 21, a=15.0005(1), c=21.8165(2) \AA, V=4251.35(6) \AA^{-3}, Z=6, D_{\mathrm{x}}=1.114$ g cm${ }^{-3}$, crystal dimensions: $0.25 \times 0.30 \times 0.30 \mathrm{~mm}, T=160 \mathrm{~K}$, Nonius KappaCCD diffractometer, Mo-K $\alpha$ radiation, $\lambda=0.71073 \AA, \mu=0.0976 \mathrm{~mm}^{-1}, \phi$ and $\omega$ scans, $\theta_{\max }=$ $26^{\circ}, 85545$ measured reflections of which 5578 were unique and 4561 had $I>2 \sigma(I)$, no absorption correction. The structure was solved by the direct methods routine of SIR $92^{1}$ and refined using SHELXL97 ${ }^{3}$ All non-hydrogen atoms were refined anisotropically. The amide and carboxyl $\mathrm{H}$-atoms were refined isotropically and the $\mathrm{H}$-atom of the carboxyl group is disordered equally over the two $\mathrm{O}$-atoms of this group. All remaining $\mathrm{H}$-atoms were placed in geometrically calculated positions and refined using a riding model with $\mathrm{U}_{\mathrm{iso}}(\mathrm{H})=1.2 \mathrm{U}_{\mathrm{eq}}(\mathrm{C})$ $\left(1.5 \mathrm{U}_{\text {eq }}(\mathrm{C})\right.$ for the methyl groups). The refinement on $F^{2}$ of 369 parameters using 5573 reflections gave $R(F)\left[I>2 \sigma(I)\right.$ data] $=0.0368, w R\left(F^{2}\right)$ [all data] $=0.0939, \mathrm{~S}=1.049, \Delta \rho_{\max }$ $=0.18 \mathrm{e} \AA^{-3}$. A correction for secondary extinction was applied. The enantiomorph was chosen using the known $(3 S, 4 R)$-configuration of the $\beta$-lactam ring. The iso-butyl group of the molecule is disordered over two conformations and two positions were successfully refined for each of the four $\mathrm{C}$-atoms of this group. The site occupation factors of the two conformations were refined initially and then held fixed at their optimum values of 0.6:0.4. Each unit cell contains one void centred at $(0,0,0)$ with a volume of $345 \AA^{3}$ and the voids form channels which run parallel to the $c$-axis. There was no evidence of any significant residual electron density in the void. Presumably the channels were once occupied by solvent molecules which have since evaporated, but the crystal lattice has remained stable. The $\mathrm{CO}_{2} \mathrm{H}_{(\mathrm{i}+3)}$ group is both a donor and an acceptor for intermolecular hydrogen bonds with the carboxylic acid group of a neighboring molecule, thereby forming closed loops with the graph set motif ${ }^{34}$ of $\mathrm{R}^{2}{ }_{2}(8)$. On the other hand, the carbamate $\mathrm{HN}_{(\mathrm{i}+1)}$ group forms an intermolecular hydrogen bond with the glycine $\mathrm{CO}_{(\mathrm{i}+2)}$ oxygen-atom of a different neighboring molecule. This latter interaction links the molecules into extended chains which have a graph set motif of $\mathrm{C}(8)$. The combination of all intermolecular interactions links the molecules into a threedimensional framework.

Crystal data for compound 18: $\mathrm{C}_{30} \mathrm{H}_{44} \mathrm{~N}_{2} \mathrm{O}_{3} \mathrm{Si}_{2}, M_{\mathrm{r}}=536.85$, colorless prisms, orthorhombic, space group $P 2{ }_{1}{ }_{2}{ }_{1}{ }_{1}, a=14.356(2), b=16.930(2), c=12.862(2) \AA, V=$ 3126.3(5) $\AA^{-3}, Z=4, D_{\mathrm{x}}=1.140 \mathrm{~g} \mathrm{~cm}^{-3}$, crystal dimensions: $0.35 \times 0.45 \times 0.50 \mathrm{~mm}, T=173$ $\mathrm{K}$, Rigaku AFC5R diffractometer, Mo-K $\alpha$ radiation, $\lambda=0.71073 \AA, \mu=0.144 \mathrm{~mm}^{-1}, \omega / 2 \theta$ scans, $\theta_{\max }=27.5^{\circ}$, Friedel pairs for all reflections with $\theta<25^{\circ}, 7774$ measured reflections of which 6489 were unique, no absorption correction. The structure was solved by the direct methods routine of SIR92 ${ }^{1}$ and refined using teXsan ${ }^{2}$. All non-hydrogen atoms were refined 
anisotropically. All $\mathrm{H}$-atoms were fixed in geometrically calculated positions with $\mathrm{U}_{\text {iso }}(\mathrm{H})=$ $1.2 \mathrm{U}_{\mathrm{eq}}(\mathrm{C})$. The refinement on $F$ of 336 parameters using 5583 observed reflections with $I>$ $2 \sigma(I)$ gave $R(F)=0.0373, w R(F)=0.0331, \mathrm{~S}=1.662, \Delta \rho_{\max }=0.25 \mathrm{e}^{-3}$ and an absolute structure parameter of $0.00(8)$. A correction for secondary extinction was applied. The enantiomorph was chosen using the known $S$-configuration of the benzylic stereocenter in the oxazolidine ring and was confirmed independently by the crystal-structure analysis.

Crystal data for compound 32: $\mathrm{C}_{19} \mathrm{H}_{32} \mathrm{~N}_{4} \mathrm{O}_{5}, M_{\mathrm{r}}=396.48$, colorless prisms, monoclinic, space group $P 2{ }_{1}, a=9.939(2), b=9.160(2), c=12.154(2) \AA, \beta=103.05(1)^{\circ}, V=1077.9(3)$ $\AA^{-3}, Z=2, D_{\mathrm{x}}=1.221 \mathrm{~g} \mathrm{~cm}^{-3}$, crystal dimensions: $0.18 \times 0.23 \times 0.50 \mathrm{~mm}, T=173 \mathrm{~K}$, Rigaku AFC5R diffractometer, Mo-K $\alpha$ radiation, $\lambda=0.71073 \AA, \mu=0.0889 \mathrm{~mm}^{-1}, \omega / 2 \theta$ scans, $\theta_{\max }=27.5^{\circ}, 2774$ measured reflections of which 2625 were unique, no absorption correction. The structure was solved by the direct methods routine of SHELXS97 ${ }^{4}$ and refined using teXsan ${ }^{2}$. All non-hydrogen atoms were refined anisotropically. All H-atoms were fixed in geometrically calculated positions with $\mathrm{U}_{\mathrm{iso}}(\mathrm{H})=1.2 \mathrm{U}_{\mathrm{eq}}$ (parent atom). The refinement on $F$ of 253 parameters using 2159 observed reflections with $I>2 \sigma(I)$ gave $R(F)=0.0440$, $w R(F)=0.0392, \mathrm{~S}=1.614, \Delta \rho_{\max }=0.20 \mathrm{e}^{-3}$. A correction for secondary extinction was applied. The enantiomorph was chosen using the known $S$-configuration of the stereocenter in the pyrrolidine ring. The $\mathrm{H}^{\mathrm{Z}} \mathrm{N}_{(\mathrm{i}+3)}$ forms an intermolecular hydrogen bond with the Boc $(\mathrm{C}=\mathrm{O})_{(\mathrm{i})}$ oxygen atom of a neighboring molecule. This interaction links the molecules into extended chains which run parallel to the $y$-axis and have a graph set motif ${ }^{34}$ of $\mathrm{C}(13)$. The $\mathrm{HN}_{(\mathrm{i}+1)}$ amide group forms bifurcated intermolecular hydrogen bonds. One of these interactions is with the glycinamide $\mathrm{CO}_{(\mathrm{i}+2)}$ oxygen atom of a neighboring molecule, while the other is a weak interaction with the $\beta$-lactam $\mathrm{CO}_{(\mathrm{i}+1)}$ oxygen atom of a different neighboring molecule. Considered independently, each of these interactions links the molecules into extended zig-zag chains which run parallel to the $y$-axis with graph set motifs of $\mathrm{C}(8)$ and $\mathrm{C}(5)$, respectively. The combination of all hydrogen-bonding interactions links the molecules into extended two-dimensional networks which lie parallel to the $y z$-plane.

1 Altomare, A.; Cascarano, G.; Giacovazzo, C.; Guagliardi, A.; Burla, M. C.; Polidori, G.; Camalli, M. SIR92;

J. Appl. Crystallogr. 1994, 27, 435.

2 teXsan, Single Crystal Structure Analysis Software, Version 1.10b; Molecular Structure Corporation: The Woodlands, Texas, 1999.

3 Sheldrick, G. M. SHELXL97, Program for the Refinement of Crystal Structures; University of Göttingen: Göttingen, Germany, 1997.

4 Sheldrick, G. M. SHELXS97, Program for the Solution of Crystal Structures; University of Göttingen: Göttingen, Germany, 1997. 
Table 1. Final Force Field Parameters and Physical Properties for DMSO (Calculated from Simulations and Experimental Data).

Nonbonded Parameters

\begin{tabular}{cccc}
\hline atom & charge & $\varepsilon\left(\mathrm{kcal} \mathrm{mol}^{-1}\right)$ & $\mathrm{R}^{*}$ \\
\hline $\mathrm{S}$ & 0.303 & 0.300 & 2.100
\end{tabular}

Bond Parameters

\begin{tabular}{ccc}
\hline bond & $k_{\mathrm{b}}\left(\mathrm{kcal} \mathrm{mol}^{-1} \AA^{2}\right)$ & $b_{\mathrm{o}}(\AA)$ \\
\hline $\mathrm{C}-\mathrm{S}$ & 317.0 & 1.87 \\
$\mathrm{~S}-\mathrm{O}$ & 570.0 & 1.67
\end{tabular}

Angle Parameters

\begin{tabular}{ccc}
\hline angle & $k_{\theta}\left(\mathrm{kcal} \mathrm{mol}^{-1} \mathrm{rad}^{-2}\right)$ & $\theta_{0}(\mathrm{deg})$ \\
\hline H-C-S & 46.1 & 111.3 \\
C-S-O & 80.0 & 105.5 \\
C-S-C & 63.0 & 95.0
\end{tabular}

Dihedral Parameters

\begin{tabular}{cccc}
\hline dihedral angle & $k_{\phi}\left(\mathrm{kcal} \mathrm{mol}^{-1}\right)$ & $n$ & $\delta(\mathrm{deg})$ \\
\hline $\mathrm{H}-\mathrm{C}-\mathrm{S}-\mathrm{O}^{\mathrm{g}}$ & 0.2 & 3 & 0 \\
$\mathrm{H}-\mathrm{C}-\mathrm{S}-\mathrm{C}^{\mathrm{g}}$ & 0.2 & 3 & 0
\end{tabular}

Comparison of Experimental and Simulation Results for Physical Properties of Pure DMSO

\begin{tabular}{ccc}
\hline property & calculated & experimental \\
\hline density $\left(\mathrm{g} \mathrm{cm}^{-3}\right)^{9}$ & 1.041 & 1.091 \\
$\Delta H_{\text {vap }}\left(\mathrm{kcal} \mathrm{mol}^{-1}\right)^{9}$ & 13.40 & 12.63
\end{tabular}

The molecular structure of DMSO was optimized at the Hartree-Fock level with the ab initio quantum mechanical program Gaussian $98^{1}$ using the $3-21 G(d)$ basis set. Partial atomic charges were then obtained using the RESP ${ }^{2}$ methodology with the 6-31G(d) basis set. A periodic cubic cell $(29.8 \AA$ x $29.8 \AA$ x $29.8 \AA$ ) containing 222 solvent molecules of DMSO was constructed to reproduce the density and enthalpy of vaporization (DHvap) of this liquid at $300 \mathrm{~K}$ using a compressibility value of $52.510^{-6} \mathrm{bar}^{-1}$. 3 The molecular dynamics (MD) simulations were carried out at $300 \mathrm{~K}$ using the SANDER module in AMBER 6. ${ }^{4}$ Both the temperature and the pressure were coupled to thermal and pressure baths with relaxation times of 0.2 and $0.6 \mathrm{ps}$, respectively. In a 20 ps heating phase, the temperature was gradually increased under constant-volume conditions, and the velocities were reassigned at each new temperature according to a Maxwell-Boltzmann distribution. The system was then equilibrated during $200 \mathrm{ps}$ at $300 \mathrm{~K}$ followed by a 1-ns sampling period at constant pressure 
during which system coordinates were collected every picosecond to yield an ensemble of 1000 structures for further analysis. $\mathrm{SHAKE}^{5}$ was applied to all bonds involving hydrogens and the integration time step was 1 fs. A nonbonded cutoff of $9 \AA$ was employed throughout. Density values were provided directly by the SANDER module of AMBER. $\Delta H_{\text {vap }}$ values were calculated according to the equation

$$
\Delta H_{\mathrm{vap}}=R T-E_{\mathrm{inter}}
$$

where $E_{\text {inter }}$ is the interaction energy of the system, which encompasses both the electrostatic and van der Waals components obtained directly from the SANDER output, divided by the number of molecules in each box.

1 Frisch, M. J.; Trucks, G. W.; Schlegel, H. B.; Scuseria, G. E.; Robb, M. A.; Cheeseman, J. R.; Zakrzewski, V. G.; Montgomery, J. A. Jr.; Stratmann, R. E.; Burant, J. C.; Dapprich, S.; Millam, J. M.; Daniels, A. D.; Kudin, K. N.; Strain, M. C.; Farkas, O.; Tomasi, J.; Barone, V.; Cossi, M.; Cammi, R.; Mennucci, B.; Pomelli, C.; Adamo, C.; Clifford, S.; Ochterski, J.; Petersson, G. A.; Ayala, P. Y.; Cui, Q.; Morokuma, K.; Malick, D. K.; Rabuck, A. D.; Raghavachari, K.; Foresman, J. B.; Cioslowski, J.; Ortiz, J. V.; Stefanov, B. B.; Liu, G.; Liashenko, A.; Piskorz, P.; Komaromi, I.; Gomperts, R.; Martin, R. L.; Fox, D. J.; Keith, T.; AlLaham, M. A.; Peng, C. Y.; Nanayakkara, A.; Gonzalez, C.; Challacombe, M.; Gill, P. M. W.; Johnson, B. G.; Chen, W.; Wong, M. W.; Andres, J. L.; Head-Gordon, M.; Replogle, E. S.; Pople, J. A. Gaussian 98, Gaussian, Inc.: Pittsburgh, PA, 1998.

2 Bayly, C. I.; Cieplak, P.; Cornell, W. D.; Kollman, P. A. A well-behaved electrostatic potential based method using charge restraints for deriving atomic charges: the RESP model. J. Phys. Chem. 1993, 97, 10269-10280.

3 Lide, D. R. (1997) CRC Handbook of Chemistry and Physics, CRC Press, Boca Raton, FL.

4 Case, D. A.; Pearlman, D. A.; Caldwell, J. W.; Cheatham, T. E., III; Ross, W. S.; Simmerling, C. L.; Darden, T. A.; Merz, K. M.; Stanton, R. V.; Cheng, A. L.; Vincent, J. J.; Crowley, M.; Tsui, V.; Radmer, R. J.; Duan, Y.; Pitera, J.; Massova, I.; Seibel, G. L.; Singh, U. C.; Weiner, P. K.; Kollman, P. A. AMBER 6; University of California, San Francisco, 1999.

5 Ryckaert, J. P.; Ciccoti, G.; Berendsen, H. J. C. Numerical integration of the Cartesian equations of motion of a system with constraints: molecular dynamics of $n$-alkanes. J. Comput. Phys. 1977, 23, 327-341.

8 Strader, M. L.; Feller, S. E. J. Phys. Chem. A 2002, 106, 1074-1080.

9 Vishnyakov, A.; Lyubartsev, A. P.; Laaksonen, A. J. Phys. Chem. A 2001, 105, 1702-1710. 
Table S1. Experimental (NMR) and calculated interprotonic distances.

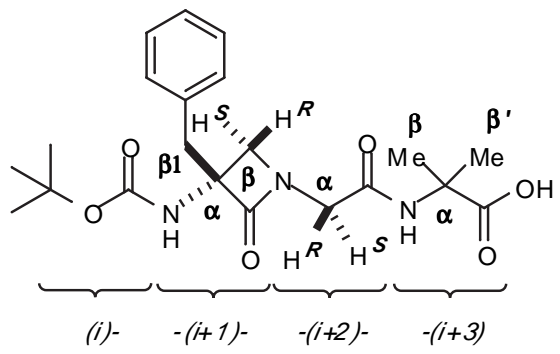

6

\begin{tabular}{cccc||cccc}
\hline $\mathrm{H}$ atom & $\mathrm{H}$ atom & $\mathrm{NOE}^{[\mathrm{a}]}$ & $\mathrm{r}^{[\mathrm{b}]}$ & $\mathrm{H}$ atom & $\mathrm{H}$ atom & $\mathrm{NOE}^{[\mathrm{a}]}$ & $\mathrm{r}^{[\mathrm{b}]}$ \\
\hline $\mathrm{HN}_{(\mathrm{i}+3)}$ & $\mathrm{H}^{\mathrm{S}} \alpha_{(\mathrm{i}+2)}$ & $\mathrm{m}$ & 3.23 & $\mathrm{H}-o-\mathrm{Ph}_{(\mathrm{i}+1)}$ & $\mathrm{H}^{\mathrm{R}} \beta 1_{(\mathrm{i}+1)}$ & $\mathrm{s}$ & 2.40 \\
$\mathrm{HN}_{(\mathrm{i}+3)}$ & $\mathrm{H}^{\mathrm{R}} \alpha_{(\mathrm{i}+2)}$ & $\mathrm{w}$ & 3.71 & $\mathrm{H}-o-\mathrm{Ph}_{(\mathrm{i}+1)}$ & $\mathrm{H}^{\mathrm{S}} \beta 1_{(\mathrm{i}+1)}$ & $\mathrm{s}$ & 2.40 \\
$\mathrm{HN}_{(\mathrm{i}+3)}$ & $\mathrm{H}^{\mathrm{S}} \beta_{(i+1)}$ & $\mathrm{m}$ & 2.87 & $\mathrm{H}^{\mathrm{S}} \beta 1_{(i+1)}$ & $\mathrm{H}^{\mathrm{R}} \beta_{(\mathrm{i}+1)}$ & $\mathrm{w}$ & 3.56 \\
$\mathrm{HN}_{(\mathrm{i}+1)}$ & $\mathrm{H}^{\mathrm{S}} \beta_{(\mathrm{i}+1)}$ & $\mathrm{w}$ & 3.55 & & & & \\
\hline
\end{tabular}

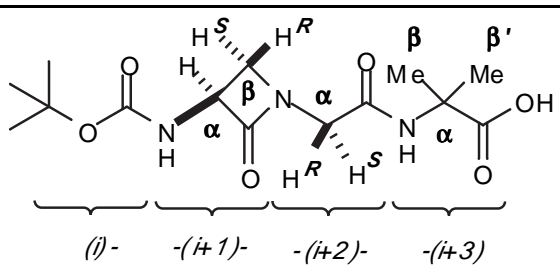

8

\begin{tabular}{cccc||cccc}
\hline $\mathrm{H}$ atom & $\mathrm{H}$ atom & $\mathrm{NOE}^{[\mathrm{a}]}$ & $\mathrm{r}^{[\mathrm{b}]}$ & $\mathrm{H}$ atom & $\mathrm{H}$ atom & $\mathrm{NOE}^{[\mathrm{a}]}$ & $\mathrm{r}^{[\mathrm{b}]}$ \\
\hline $\mathrm{HN}_{(\mathrm{i}+3)}$ & $\mathrm{H}^{\mathrm{S}} \beta_{(\mathrm{i}+1)}$ & $\mathrm{w}$ & 5.02 & $\mathrm{HN}_{(\mathrm{i}+1)}$ & $\mathrm{H} \alpha_{(\mathrm{i}+1)}$ & $\mathrm{m}$ & 2.84 \\
$\mathrm{HN}_{(\mathrm{i}+3)}$ & $\mathrm{H}^{\mathrm{R}} \beta_{(\mathrm{i}+1)}$ & $\mathrm{m}$ & 3.36 & $\mathrm{HN}_{(\mathrm{i}+1)}$ & $\mathrm{H}^{\mathrm{R}} \beta_{(\mathrm{i}+1)}$ & $\mathrm{w}$ & 3.53 \\
$\mathrm{HN}_{(\mathrm{i}+3)}$ & $\mathrm{H}^{\mathrm{S}} \alpha_{(\mathrm{i}+2)}$ & $\mathrm{w}$ & 3.65 & $\mathrm{H} \alpha_{(\mathrm{i}+1)}$ & $\mathrm{H}^{\mathrm{S}} \beta_{(\mathrm{i}+1)}$ & $\mathrm{s}$ & 2.46 \\
$\mathrm{HN}_{(\mathrm{i}+3)}$ & $\mathrm{H}^{\mathrm{R}} \alpha_{(\mathrm{i}+2)}$ & $\mathrm{w}$ & 3.62 & $\mathrm{H} \alpha_{(\mathrm{i}+1)}$ & $\mathrm{H}^{\mathrm{R}} \beta_{(\mathrm{i}+1)}$ & $\mathrm{w}$ & 3.64 \\
\hline
\end{tabular}




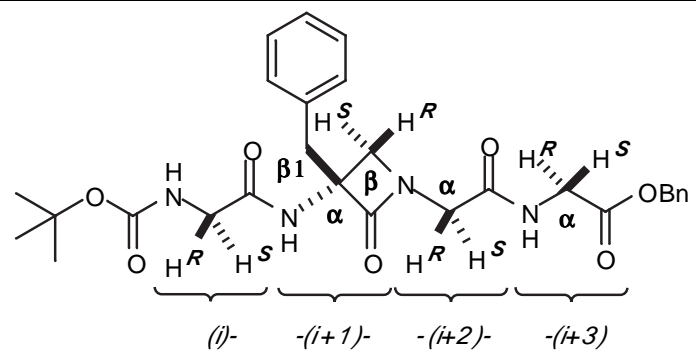

9

\begin{tabular}{cccc||cccc}
\hline $\mathrm{H}$ atom & $\mathrm{H}$ atom & $\mathrm{NOE}^{[\mathrm{a}]}$ & $\mathrm{r}^{[\mathrm{b}]}$ & $\mathrm{H}$ atom & $\mathrm{H}$ atom & $\mathrm{NOE}^{[\mathrm{a}]}$ & $\mathrm{r}^{[\mathrm{b}]}$ \\
\hline $\mathrm{HN}_{(\mathrm{i}+3)}$ & $\mathrm{H}^{\mathrm{S}} \alpha_{(\mathrm{i}+2)}$ & $\mathrm{w}$ & 3.85 & $\mathrm{HN}_{(\mathrm{i}+1)}$ & $\mathrm{H}^{\mathrm{S}} \beta_{(\mathrm{i}+1)}$ & $\mathrm{w}$ & 4.47 \\
$\mathrm{HN}_{(\mathrm{i}+3)}$ & $\mathrm{H}^{\mathrm{S}} \beta_{(\mathrm{i}+1)}$ & $\mathrm{s}$ & 2.63 & $\mathrm{HN}_{(\mathrm{i}+1)}$ & $\mathrm{HN}_{(\mathrm{i})}$ & $\mathrm{m}$ & 3.13 \\
$\mathrm{HN}_{(\mathrm{i}+3)}$ & $\mathrm{H}^{\mathrm{R}} \alpha_{(\mathrm{i}+3)}$ & $\mathrm{s}$ & 2.63 & $\mathrm{HN}_{(\mathrm{i})}$ & $\mathrm{H}^{\mathrm{R}} \alpha_{(\mathrm{i})}$ & $\mathrm{m}$ & 2.98 \\
$\mathrm{HN}_{(\mathrm{i}+1)}$ & $\mathrm{H}^{\mathrm{R}} \alpha_{(\mathrm{i})}$ & $\mathrm{m}$ & 3.14 & $\mathrm{HN}_{(\mathrm{i})}$ & $\mathrm{H}^{\mathrm{S}} \alpha_{(\mathrm{i})}$ & $\mathrm{m}$ & 3.46 \\
$\mathrm{HN}_{(\mathrm{i}+1)}$ & $\mathrm{H}^{\mathrm{S}} \alpha_{(\mathrm{i})}$ & $\mathrm{m}$ & 3.28 & $\mathrm{H}^{\mathrm{S}} \beta_{(\mathrm{i}+1)}$ & $\mathrm{H}^{\mathrm{S}} \alpha_{(\mathrm{i}+2)}$ & $\mathrm{w}$ & 4.31 \\
\hline
\end{tabular}

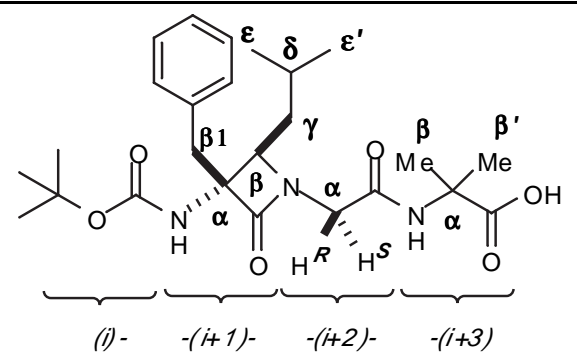

10

\begin{tabular}{cccc||cccc}
\hline $\mathrm{H}$ atom & $\mathrm{H}$ atom & $\mathrm{NOE}^{[\mathrm{a}]}$ & $\mathrm{r}^{[\mathrm{b}]}$ & $\mathrm{H}$ atom & $\mathrm{H}$ atom & $\mathrm{NOE}^{[\mathrm{a}]}$ & $\mathrm{r}^{[\mathrm{b}]}$ \\
\hline $\mathrm{HN}_{(\mathrm{i}+3)}$ & $\mathrm{H}^{\mathrm{S}} \alpha_{(i+2)}$ & $\mathrm{w}$ & 4.48 & $\mathrm{H} \beta_{(\mathrm{i}+1)}$ & $\mathrm{H} \delta_{(i+1)}$ & $\mathrm{s}$ & 2.10 \\
$\mathrm{HN}_{(\mathrm{i}+3)}$ & $\mathrm{H}^{\mathrm{S}} \beta_{(i+1)}$ & $\mathrm{m}$ & 3.01 & $\mathrm{H}^{\mathrm{S}} \alpha_{(\mathrm{i}+2)}$ & $\mathrm{H} \delta_{(\mathrm{i}+1)}$ & $\mathrm{s}$ & 2.59 \\
$\mathrm{HN}_{(\mathrm{i}+3)}$ & $\mathrm{H}^{\mathrm{R}} \alpha_{(i+2)}$ & $\mathrm{w}$ & 3.60 & $\mathrm{H}^{\mathrm{S}} \beta 1_{(i+1)}$ & $\mathrm{H} \delta_{(i+1)}$ & $\mathrm{s}$ & 2.29 \\
$\mathrm{HN}_{(\mathrm{i}+1)}$ & $\mathrm{H}^{\mathrm{R}} \beta 1_{(i+1)}$ & $\mathrm{w}$ & 3.78 & $\mathrm{H}^{\mathrm{R}} \beta 1_{(\mathrm{i}+1)}$ & $\mathrm{H} \delta_{(i+1)}$ & $\mathrm{m}$ & 2.72 \\
$\mathrm{HN}_{(\mathrm{i}+1)}$ & $\mathrm{H}^{\mathrm{S}} \beta 1_{(i+1)}$ & $\mathrm{s}$ & 2.58 & & & & \\
\hline
\end{tabular}




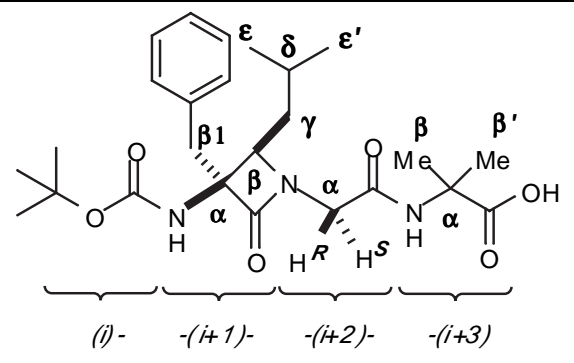

11

\begin{tabular}{cccc||cccc}
\hline $\mathrm{H}$ atom & $\mathrm{H}$ atom & $\mathrm{NOE}^{[\mathrm{a}]}$ & $\mathrm{r}^{[\mathrm{b}]}$ & $\mathrm{H}$ atom & $\mathrm{H}$ atom & $\mathrm{NOE}^{[\mathrm{a}]}$ & $\mathrm{r}^{[\mathrm{b}]}$ \\
\hline $\mathrm{HN}_{(\mathrm{i}+3)}$ & $\mathrm{H}^{\mathrm{S}} \alpha_{(\mathrm{i}+2)}$ & $\mathrm{w}$ & 3.85 & $\mathrm{H} \beta_{(\mathrm{i}+1)}$ & $\mathrm{H}^{\mathrm{R}} \beta 1_{(i+1)}$ & $\mathrm{m}$ & 3.07 \\
$\mathrm{HN}_{(\mathrm{i}+3)}$ & $\mathrm{H}^{\mathrm{R}} \alpha_{(i+2)}$ & $\mathrm{w}$ & 3.89 & $\mathrm{H} \beta_{(\mathrm{i}+1)}$ & $\mathrm{H}^{\mathrm{S}} \beta 1_{(i+1)}$ & $\mathrm{m}$ & 3.18 \\
$\mathrm{HN}_{(\mathrm{i}+1)}$ & $\mathrm{H}^{\mathrm{R}} \beta 1_{(\mathrm{i}+1)}$ & $\mathrm{m}$ & 3.36 & $\mathrm{H}-o-\mathrm{Ph}_{(\mathrm{i}+1)}$ & $\mathrm{H}^{\mathrm{R}} \beta 1_{(i+1)}$ & $\mathrm{m}$ & 2.92 \\
$\mathrm{HN}_{(\mathrm{i}+1)}$ & $\mathrm{H}^{\mathrm{S}} \beta 1_{(i+1)}$ & $\mathrm{w}$ & 3.65 & $\mathrm{H}-o-\mathrm{Ph}_{(i+1)}$ & $\mathrm{H}^{\mathrm{S}} \beta 1_{(i+1)}$ & $\mathrm{m}$ & 2.94 \\
$\mathrm{HN}_{(\mathrm{i}+3)}$ & $\mathrm{H}^{\mathrm{S}} \gamma_{(i+1)}$ & $\mathrm{w}$ & 4.16 & $\mathrm{HN}_{(\mathrm{i}+3)}$ & $\mathrm{H}^{\mathrm{S}} \gamma_{(i+1)}$ & $\mathrm{w}$ & 3.83 \\
\hline
\end{tabular}

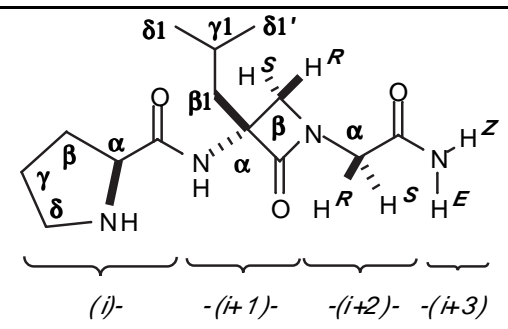

13

\begin{tabular}{cccc||cccc}
\hline $\mathrm{H}$ atom & $\mathrm{H}$ atom & $\mathrm{NOE}^{[\mathrm{a}]}$ & $\mathrm{r}^{[\mathrm{b}]}$ & $\mathrm{H}$ atom & $\mathrm{H}$ atom & $\mathrm{NOE}^{[\mathrm{a}]}$ & $\mathrm{r}^{[\mathrm{b}]}$ \\
\hline $\mathrm{H}^{\mathrm{E}} \mathrm{N}_{(\mathrm{i}+3)}$ & $\mathrm{H}^{\mathrm{R}} \alpha_{(\mathrm{i}+2)}$ & $\mathrm{m}$ & 3.15 & $\mathrm{H}^{\mathrm{Z}} \mathrm{N}_{(\mathrm{i}+3)}$ & $\mathrm{H}^{\mathrm{S}} \alpha_{(\mathrm{i}+2)}$ & $\mathrm{m}$ & 3.14 \\
$\mathrm{H}^{\mathrm{E}} \mathrm{N}_{(\mathrm{i}+3)}$ & $\mathrm{H}^{\mathrm{S}} \alpha_{(\mathrm{i}+2)}$ & $\mathrm{m}$ & 3.06 & $\mathrm{H}^{\mathrm{Z}} \mathrm{N}_{(\mathrm{i}+3)}$ & $\mathrm{H}^{\mathrm{S}} \beta_{(\mathrm{i}+1)}$ & $\mathrm{m}$ & 3.01 \\
$\mathrm{H}^{\mathrm{E}} \mathrm{N}_{(\mathrm{i}+3)}$ & $\mathrm{H}^{\mathrm{S}} \beta_{(i+1)}$ & $\mathrm{m}$ & 2.88 & $\mathrm{HN}_{(\mathrm{i}+1)}$ & $\mathrm{H}^{\mathrm{S}} \beta_{(\mathrm{i}+1)}$ & $\mathrm{w}$ & 4.25 \\
$\mathrm{H}^{\mathrm{Z}} \mathrm{N}_{(\mathrm{i}+3)}$ & $\mathrm{H}^{\mathrm{R}} \alpha_{(i+2)}$ & $\mathrm{m}$ & 3.26 & $\mathrm{HN}_{(\mathrm{i}+1)}$ & $\mathrm{H} \alpha_{(\mathrm{i})}$ & $\mathrm{m}$ & 3.22 \\
\hline
\end{tabular}

[a] Upper bound NOEs set to $2.7 \AA$ for "strong", $3.3 \AA$ for "medium" and $5.0 \AA$ for "weak", according to customary. ${ }^{1}$

${ }^{[b]}$ Experimental interprotonic distances $(\AA)$ from the integration of 500-ms NOESY $(500 \mathrm{MHz})$ spectrum of $\beta$ lactam peptidomimetic 6 in $\mathrm{DMSO}_{\mathrm{d} 6}$, at $300^{\circ} \mathrm{K}$.

1 Williamson, M. P.; Havel, T. F.; Wüthrich, J. Mol Biol. 1985, 182, 295-315. 